\title{
The direct oxygen abundances of metal-rich galaxies derived from electron temperature ${ }^{\star}$
}

\author{
Y. C. Liang ${ }^{1,2}$, F. Hammer ${ }^{2}$, S. Y. Yin ${ }^{1,3,4}$, H. Flores ${ }^{2}$, M. Rodrigues ${ }^{2}$, and Y. B. Yang ${ }^{2,1}$ \\ ${ }^{1}$ National Astronomical Observatories, Chinese Academy of Sciences, 20A Datun Road, Chaoyang District, Beijing 100012, \\ PR China \\ e-mail: ycliang@bao.ac.cn \\ 2 GEPI, Observatoire de Paris-Meudon, 92195 Meudon, France \\ 3 Department of Physics, Hebei Normal University, Shijiazhuang 050016, PR China \\ 4 Department of Physics, Harbin University, Haerbin 150086, PR China
}

Received 8 March 2007 / Accepted 17 July 2007

\section{ABSTRACT}

\begin{abstract}
Context. Direct measurement of oxygen abundance for metal-rich galaxies from electron temperature is difficult or impossible since temperature-sensitive auroral lines generally become too weak to be measured.

Aims. We aim to derive the electron temperature $\left(T_{\mathrm{e}}\right)$ in the gas of metal-rich star-forming galaxies, which can be obtained from their ratios of auroral lines [O II] $\lambda \lambda 7320,7330$ to nebular lines [O II] $\lambda 3727$, in order to establish a more robust massmetallicity relationship, and compare the $T_{\mathrm{e}}$-based $(\mathrm{O} / \mathrm{H})$ abundances with those from empirical strong-line calibrations, such as $R_{23}(=([\mathrm{O}$ II $] \lambda 3727+[\mathrm{O}$ III $] \lambda \lambda 4959,5007) / \mathrm{H} \beta$ ).

Methods. We obtained 27 spectra by stacking the spectra of several hundred (even several thousand) star-forming galaxies selected from the SDSS-DR4 in each of the 27 stellar mass bins from $\log \left(M_{*}\right) \sim 8.0$ to 10.6 (in units $\operatorname{of} \log \left(M_{\odot}\right)$ ). This "stack" method sufficiently improves the signal-to-noise ratio of the auroral lines [O II] $\lambda \lambda 7320,7330$. Using a two-zone model for the temperature structure, we derive the electron temperature $t_{2}$ in the low ionization region from the $[\mathrm{O} \mathrm{II}] \lambda \lambda 7320,7330 /[\mathrm{O} \mathrm{II}] \lambda 3727 \mathrm{ratio}$, and then use a relation derived by fitting $\mathrm{H}$ II region models to estimate the electron temperature $t_{3}$ in the high ionization region from $t_{2}$. Then, the direct $(\mathrm{O} / \mathrm{H})$ abundances are obtained from $t_{2}, t_{3}$ and the related line-ratios. The emission lines have been carefully corrected for dust extinction using the Balmer line ratio after correcting for the underlying stellar absorption.

Results. Combining our results with those from the literature with lower metallicities, we are able to provide a new relationship between the direct measurements of $(\mathrm{O} / \mathrm{H})$ and $R_{23}$, which still shows an upper and a lower branch with the transition around $12+\log (\mathrm{O} / \mathrm{H}) \sim 8.0-8.2$. It also shows that the empirical $R_{23}$ method will overestimate $\log (\mathrm{O} / \mathrm{H})$ by 0.2 to 0.6 dex. The new metal-mass relationship of the galaxies with moderate metallicities is fitted by a linear fit $\left(12+\log (\mathrm{O} / \mathrm{H})=6.223+0.231 \times \log \left(M_{*}\right)\right)$ confirming that empirical methods significantly overestimate $(\mathrm{O} / \mathrm{H})$. We also derived their $(\mathrm{N} / \mathrm{O})$ abundance ratios on the basis of the $T_{\mathrm{e}}$ method, which are consistent with the combination of the primary and secondary components of nitrogen.

Conclusions. This study provides for the first time a method to calibrate direct $\mathrm{O} / \mathrm{H}$ abundances (from $T_{\mathrm{e}}$ ) for a large range of galaxies within a stellar mass range of $\sim 5 \times 10^{8} M_{\odot}$ to $4 \times 10^{10} M_{\odot}$.
\end{abstract}

Key words. galaxies: abundances - galaxies: evolution - galaxies: ISM - galaxies: spiral - galaxies: starburst galaxies: stellar content

\section{Introduction}

The chemical properties of stars and gas within a galaxy provide both a fossil record of its star formation history and information on its present evolutionary status. Accurate abundance measurements for the ionized gas in galaxies require the determination of electron temperature $\left(T_{\mathrm{e}}\right)$ in the gas, which is usually obtained from the ratio of auroral to nebular line intensities, such as [O III $] \lambda \lambda 4959,5007 /[\mathrm{O}$ III $] \lambda 4363$. This is generally known as the "direct $T_{\mathrm{e}}$-method" since $T_{\mathrm{e}}$ is directly inferred from observed line ratios. However, it is well known that this procedure is difficult to carry out for metal-rich galaxies since, as the metallicity increases, the electron temperature decreases (as the cooling is via metal lines), and the auroral lines eventually become too faint to measure. Instead, other strong nebular line ratios have to be used to estimate the oxygen abundances of metal-rich galaxies $(12+\log (\mathrm{O} / \mathrm{H}) \geq 8.5)$,

* Figure 1 and Table 2 are only available in electronic form at http://www . aanda.org such as the $R_{23}(=([\mathrm{O}$ II $] \lambda 3727+[\mathrm{O}$ III $] \lambda \lambda 4959,5007) / \mathrm{H} \beta)$ parameter (Pagel et al. 1979; Tremonti et al. 2004, hereafter T04, and the references therein), N2 index $(=\log ([\mathrm{N}$ II $] \lambda 6583 / \mathrm{H} \alpha))$, and some other metallicity-sensitive strong-line ratios (Kewley \& Dopita 2002; Pettini \& Pagel 2004; Stasinska 2006; Liang et al. 2006; Yin et al. 2007a). Pilyugin (2001a,b) and Pilyugin $\&$ Thuan (2005) also suggested their $P$-method to estimate the $(\mathrm{O} / \mathrm{H})$ abundances from the $R_{23}$ and $P(=[\mathrm{O} \mathrm{III}] /([\mathrm{O} \mathrm{II}]+$ [O III])) parameters.

However, these "indirect" calibrations may produce some errors in metallicity estimates. For example, the double-valued problem of $R_{23}$ for oxygen abundances, with the transition occurring near $12+\log (\mathrm{O} / \mathrm{H}) \sim 8.4\left(\log R_{23} \sim 0.8\right)(\mathrm{McGaugh}$ 1991). Most of the other strong-line ratios are sensitive to the photoionization parameter in the ionized gas (Kewley \& Dopita 2002), and some of them suffer from the same double-valued problem as $R_{23}$.

Researchers continuously try to obtain the "direct" $T_{\mathrm{e}}$-based oxygen abundances for the metal-rich galactic gas, though 
there are some disagreements (Stasinska 2005). For example, Kennicutt et al. (2003, hereafter KBG03), Bresolin et al. (2004, 2005), Bresolin (2007) and Garnett et al. (2004a,b) obtained the oxygen abundances from $T_{\mathrm{e}}$ for a handful of $\mathrm{H}$ II regions in M101 and M51 with $12+\log (\mathrm{O} / \mathrm{H})>8.4$ by using high quality spectra taken from the Very Large Telescope (VLT), Multiple Mirror Telescope (MMT) or Keck, 4-8 m telescopes. Pilyugin et al. (2006) derived a flux-flux(ff)-relation to estimate the [O III] $\lambda 4363 /\left[\mathrm{O}\right.$ III] $\lambda 5007$ ratio from the obtained $R_{23}$ by using a sample of $48 \mathrm{H}$ II regions with moderate metallicities. However, all these impressive progresses are only based on individual $\mathrm{H}$ II regions. It is very difficult to measure the temperature-sensitive auroral lines from the integrated spectra of metal-rich galaxies, hence derive their "direct" metallicities.

The wonderful Sloan Digital Sky Survey (SDSS) database allows this to be realized. It provides high quality spectra covering 3800-9200 $\AA$ with moderate resolution $(\sim 3 \AA)$ for a large number of galaxies at different given oxygen abundances or stellar masses. It makes it possible for us to measure the temperature-sensitive auroral lines [O II] $\lambda \lambda 7320,7330$ from the integrated light of metal-rich galaxies, which are usually the strongest auroral lines in the measured optical spectra of the metal-rich galactic gas. To do so, we combine the spectra of several hundred (even several thousand) galaxies in each of the different mass bins (see Sect. 2 for details). This "stack" method significantly improve the signal-to-noise $(\mathrm{S} / \mathrm{N})$ ratio of the spectra, which makes it possible to use [O II $] \lambda \lambda 7320,7330 /[\mathrm{O}$ II $] \lambda 3727$ ratios to derive their electron temperature $T_{\mathrm{e}}$ in the low ionization region $\left(t_{2}\right)$, then to obtain their "direct" $(\mathrm{O} / \mathrm{H})$ abundances. This is probably the first result concerning the derivation of "direct" $(\mathrm{O} / \mathrm{H})$ abundances from $T_{\mathrm{e}}$ from the integrated light of metal-rich star-forming galaxies.

This paper is organized as follows. The sample selection criteria and spectral combination are described in Sect. 2. The corrections for stellar absorption and flux measurements are described in Sect. 3. In Sect. 4, we re-calibrate the relations of the $(\mathrm{O} / \mathrm{H})$ abundances versus stellar masses and $R_{23}$ parameters for star-forming galaxies on the basis of the SDSS-DR4 database. The determinations of the oxygen abundances from $T_{\mathrm{e}}$ are presented in Sect. 5. In Sect. 6, we compare the $T_{\mathrm{e}}$-based $(\mathrm{O} / \mathrm{H})$ abundances with those derived from other empirical strongline methods, such as $R_{23}, P$ and N2, and derive a new relationship between $(\mathrm{O} / \mathrm{H})_{T_{\mathrm{e}}}$ and $R_{23}$. In Sect. 7, we obtain the $\log (\mathrm{N} / \mathrm{O})$ abundance ratios of these combined galaxies. The discussions are given in Sect. 8. We conclude the paper in Sect. 9. Throughout this paper, the stellar masses of galaxies $M_{*}$ are in units of solar mass $M_{\odot}$. [O II] $\lambda \lambda 7320,7330$ can also be given as one single line [O II] $\lambda 7325$, so both of these two descriptions will appear in the text.

\section{Sample selection and spectral combination}

We aim to measure the electron temperatures, hence the $T_{\mathrm{e}}$-based $(\mathrm{O} / \mathrm{H})$ abundances, for massive galaxies. [O II] $] \lambda \lambda 7320,7330$ lines are usually the strongest auroral lines in the measured optical spectra of star-forming galaxies (Bresolin et al. 2005), thus we focus on the $T_{\mathrm{e}}$ derived from the line-ratio of $[\mathrm{O}$ II $] \lambda \lambda 7320,7330 /[\mathrm{O}$ II $] \lambda 3727$. To increase the $\mathrm{S} / \mathrm{N}$ ratio of the spectra, we combine the spectra of several hundred (even several thousand) galaxies selected following the criteria described below, which makes it possible to measure [O II $] \lambda \lambda 7320,7330$ auroral lines for the massive galaxies up to $\log \left(M_{*}\right)=10.6$.
Firstly, we select $\sim 70000$ star-forming galaxies with $8.0<$ $\log \left(M_{*}\right)<10.6$ from SDSS-DR4 by following their stellar masses and metallicities estimated by the MPA/JHU group ${ }^{1}$ (T04; Kauffmann et al. 2003; Brinchmann et al. 2004; also see Liang et al. 2006, for the criteria for star-forming galaxies). This criterion allows us to select all the star-forming galaxies with available estimates of stellar masses and metallicities (above the required $\mathrm{S} / \mathrm{N}$ ratios) from the SDSS-DR4, but with stellar masses smaller than $4 \times 10^{10} M_{\odot}$. This mass limit is caused by the difficulty in obtaining measurements of [O II] $\lambda \lambda 7320,7330$ auroral lines for the most massive galaxies.

Secondly, we divide these galaxies into 27 bins with different stellar masses from $\log \left(M_{*}\right)=8$ to 10.6 . The mass bins and the total numbers of the sample galaxies in the bins are given in Cols. 3 and 5 in Table 1, respectively.

Thirdly, in each bin, we select about half of the galaxies with stronger emission lines as working samples, which allows us to obtain [O II] $\lambda \lambda 7320,7330$ lines at a high $\mathrm{S} / \mathrm{N}$ ratio for the galaxies with stellar masses up to $\log \left(M_{*}\right)=10.6$. Namely, we select the half of the galaxies having equivalent width values of [O II] $] 3727, W(\mathrm{O}$ II $)$, larger than the mean value of the whole sample of galaxies in each bin for the case of $\log \left(M_{*}\right)<10.0$, and larger than $30 \AA$ for the case of $\log \left(M_{*}\right) \geq 10.0$. The $W(\mathrm{O}$ II $)$ of the selected galaxies and the mean $W(\mathrm{O} \text { II })_{\text {mean }}(\mathrm{Col} .4$ in Table 1, given as the actual $E W([\mathrm{O}$ II] $)$ ) in each bin were gathered or calculated from the measurements published by the MPA/JHU group. The numbers of the selected samples in the bins are given in Col. 6 in Table 1. The selected sample for this study finally consists of 23608 galaxies. In the following (see Sect. 4), we will show that the above criterion creates almost no bias in estimating the mass-metallicity relationship.

Fourthly, the hundred (even several thousand) spectra of the sample galaxies in each bin are combined to be one single spectrum, which has high $\mathrm{S} / \mathrm{N}$ ratio ( $\sim 0$ at continuum) and sufficiently strong [O II $] \lambda \lambda 7320,7330$ lines for measuring. Figure 1 shows all the combined spectra at rest-frame in 27 mass bins, and the small figure in each panel shows the auroral lines [O II] $\lambda \lambda 7320,7330$ region. The basic data of the combined spectra are given in Table 1.

These combined spectra may provide a good representation of the integrated light of the real galaxies in a given stellar mass range. Obviously, they gather more light from more individual $\mathrm{H}$ II regions of the galaxies with similar properties, e.g., the similar stellar masses. However, one may wonder whether stacked spectra may keep the information that underlines the real physics of each of the individual galaxies. Indeed the intrinsic property of this is similar to what happens when studying the global properties of an individual galaxy: it is a mix, mainly of the $\mathrm{H}$ II regions of the galaxy. Here we simply sum up a much larger number of $\mathrm{H}$ II regions in a large number of galaxies, and assume that the resulting properties are near the average properties of each individual galaxy. In Sect. 4 we will verify that this assumption indeed applies for the empirical determination of $\mathrm{O} / \mathrm{H}$ (through the so-called $R_{23}$ method), and the stacking method will be discussed further in Sect. 8.2.

From our integrated-light spectra, it is still almost impossible to measure other temperature-sensitive emission-lines [N II] 25755 , [S II] 44072 . And the SDSS spectrum does not cover [S III] $\lambda \lambda 9069,9532$ though it is possible to measure the [S III] $\lambda 6312$ emission-line for the sample with $\log \left(M_{*}\right)<9.6$. Thus it is impossible to obtain directly the $T([\mathrm{~S} \mathrm{III}])$ from the [S III] ratio and the $T([\mathrm{~N} \mathrm{II}])$ from the $[\mathrm{N} \mathrm{II}]$ ratio for these

\footnotetext{
1 http://www .mpa-garching.mpg.de/SDSS/
} 
Table 1. The basic properties of the 27 combined spectra. Columns (1)-(6) refer to the counting number, the label for the mass bin (marked in Fig. 1), the mass bin, the average $E W([\mathrm{O} \mathrm{II}] \lambda 3727)$ of the sample galaxies in each bin, the total number of the galaxies, and the number of the selected spectra to be combined (about half) in each bin, respectively.

\begin{tabular}{llllrr}
\hline \hline$(1)$ & $(2)$ & $(3)$ & $(4)$ & $(5)$ & $\begin{array}{r}(6) \\
\text { No. Label }\end{array}$ \\
& & $\begin{array}{l}\text { Mass bin } \\
\left(\log M_{*}\right)\end{array}$ & $\begin{array}{l}E W([\mathrm{O} \text { II }]) \\
\text { average }(\AA)\end{array}$ & $\begin{array}{r}\text { Total } \\
\text { number }\end{array}$ & $\begin{array}{r}\text { nelected } \\
\text { number }\end{array}$ \\
\hline 1 & $\mathrm{~m} 87$ & $<8.7$ & -78.72 & 378 & 149 \\
2 & $\mathrm{~m} 871$ & $8.7-8.8$ & -73.73 & 339 & 158 \\
3 & $\mathrm{~m} 881$ & $8.8-8.9$ & -71.53 & 533 & 228 \\
4 & $\mathrm{~m} 891$ & $8.9-9.0$ & -65.51 & 797 & 341 \\
5 & $\mathrm{~m} 901$ & $9.0-9.1$ & -62.24 & 1227 & 533 \\
6 & $\mathrm{~m} 911$ & $9.1-9.2$ & -60.42 & 1470 & 593 \\
7 & $\mathrm{~m} 921$ & $9.2-9.3$ & -57.76 & 1885 & 766 \\
8 & $\mathrm{~m} 931$ & $9.3-9.4$ & -55.02 & 2428 & 1029 \\
9 & $\mathrm{~m} 9405$ & $9.40-9.45$ & -52.80 & 1329 & 552 \\
10 & $\mathrm{~m} 94505$ & $9.45-9.50$ & -51.31 & 1478 & 627 \\
11 & $\mathrm{~m} 9505$ & $9.50-9.55$ & -50.41 & 1670 & 705 \\
12 & $\mathrm{~m} 95505$ & $9.55-9.60$ & -48.14 & 1746 & 751 \\
13 & $\mathrm{~m} 9605$ & $9.60-9.65$ & -46.58 & 1920 & 814 \\
14 & $\mathrm{~m} 96505$ & $9.65-9.70$ & -45.11 & 2001 & 837 \\
15 & $\mathrm{~m} 9705$ & $9.70-9.75$ & -43.72 & 2156 & 906 \\
16 & $\mathrm{~m} 97505$ & $9.75-9.80$ & -42.17 & 2262 & 951 \\
17 & $\mathrm{~m} 9805$ & $9.80-9.85$ & -40.21 & 2428 & 1010 \\
18 & $\mathrm{~m} 98505$ & $9.85-9.90$ & -38.31 & 2841 & 1020 \\
19 & $\mathrm{~m} 9905$ & $9.90-9.95$ & -35.94 & 2552 & 1025 \\
20 & $\mathrm{~m} 99505$ & $9.95-10.0$ & -33.59 & 2605 & 1048 \\
21 & $\mathrm{~m} 10005$ & $10.0-10.05$ & -33.22 & 2750 & 1076 \\
22 & $\mathrm{~m} 100505$ & $10.05-10.10$ & -31.19 & 2814 & 1120 \\
23 & $\mathrm{~m} 1011$ & $10.10-10.20$ & $-29.12^{a}$ & 5860 & 2145 \\
24 & $\mathrm{~m} 1021$ & $10.20-10.30$ & $-26.28^{a}$ & 6004 & 1813 \\
25 & $\mathrm{~m} 1031$ & $10.30-10.40$ & $-23.79^{a}$ & 6084 & 1417 \\
26 & $\mathrm{~m} 1041$ & $10.40-10.50$ & $-22.18^{a}$ & 5973 & 1148 \\
27 & $\mathrm{~m} 1051$ & $10.50-10.60$ & $-20.72^{a}$ & 5341 & 846 \\
\hline
\end{tabular}

${ }^{a}$ For the massive galaxies with $\log \left(M_{*}\right)>10.10$, we select those with stronger [O II] emission-lines with $E W([\mathrm{O}$ II $])<-30 \AA$ for spectral combination.

sample galaxies. In a two-zone model of temperature structure, $T\left(\mathrm{~N}^{+}\right)$is equal to $T\left(\mathrm{O}^{+}\right)$and it is acceptable to omit $T\left(\mathrm{~S}^{+2}\right)$ since it represents an intermediate-ionization zone.

\section{Correction for stellar absorption and extinction}

The Balmer emission lines should be corrected carefully by the underlying stellar absorption before measuring their fluxes properly. This correction dominates the main uncertainties for the flux measurements of the Balmer lines in this study since the $\mathrm{S} / \mathrm{N}$ ratios of the combined spectra are quite high.

\subsection{Stellar absorption correction}

The methodology used here is fitting an observed spectrum, including its continuum and absorption lines, with a linear combination of simple stellar populations (SSP) generated using the population synthesis code of Bruzual \& Charlot (2003, BC03), GALAXEV. The BC03 models incorporate an empirical spectral library with a wavelength coverage (3200-9500 $\AA$ ) and spectral resolution $(\sim 3 \AA)$ that is well matched to that of the SDSS data. We use 10 templates of SSP of BC03 with different ages $(0.005$, $0.025,0.1,0.2,0.6,0.9,1.4,2.5,5,10 \mathrm{Gyr})$ at solar metallicity that are obtained by using the stellar evolution tracks of the "Padova 1994 library", and the Chabrier's IMF (Chabrier 2003).

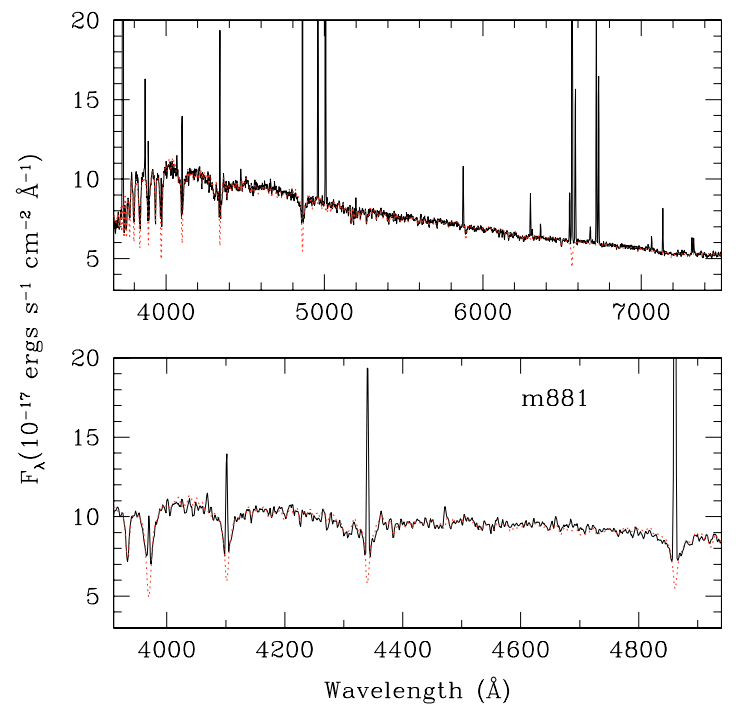

Fig. 2. The fitting on the continuum and stellar absorption for one example spectrum $\mathrm{m} 881$. The top panel is for the spectral range from 3670 to $7505 \AA$, and the bottom panel is for the detailed fitting from Ca H K lines to $\mathrm{H} \beta$ (from 3910 to $4940 \AA$ ). The solid lines indicate the observed spectrum, and the dotted lines give the fittings by using the SSP of BC03 and the program STARLIGHT (see Sect. 3.1). The top and bottom plots are made by using the same data and model.

These 10 templates are a sub-sample of the 39 templates used by T04 to fit the spectra of the SDSS galaxies, which consist of three metallicities $Z=1 / 5,1,2.5 Z_{\odot}$. The 10 templates with $Z_{\odot}$ used in this study should be sufficient to correct the stellar absorption for the spectra, since the degeneracy of age and metallicity still cannot be perfectly broken.

We use the program, STARLIGHT ${ }^{2}$, developed by Cid Fernandes and colleagues, to synthesize the stellar absorptions and continua of the sample galaxies (Cid Fernandes et al. 2005, 2007; Mateus et al. 2006; Asari et al. 2007). It is a program to fit an observed spectrum $O_{\lambda}$ with a model $M_{\lambda}$ that adds up $N_{*}$ (we use 10 as mentioned above) spectral components from a pre-defined set of base spectra. Both dust extinction on the SSP templates and line-of-sight stellar motions modeled by a Gaussian distribution with dispersion $\sigma$ have been considered in the program. The Galactic extinction law of Cardelli et al. (1989, CCM) with $R_{V}=3.1$ is adopted. The fit is carried out with the Metropolis scheme, which searches for the minimum $\chi^{2}$. We show an example in Fig. 2 for the spectral fittings.

\subsection{Flux measurements and extinction correction}

We use SPLOT in the IRAF ${ }^{3}$ package to measure the fluxes of the emission-lines from the stellar-absorption subtracted spectra. Dust extinction $\left(A_{V}\right.$, at $V$-band), is estimated by using the Balmer-line ratios $\mathrm{H} \alpha / \mathrm{H} \beta, \mathrm{H} \gamma / \mathrm{H} \beta$ and $\mathrm{H} \alpha / \mathrm{H} \gamma$, and by assuming case $\mathrm{B}$ recombination with a density of $100 \mathrm{~cm}^{-3}$, a temperature of $10^{4} \mathrm{~K}$, and the predicted intrinsic $\mathrm{H} \alpha / \mathrm{H} \beta$ ratio of 2.86 and $\mathrm{H} \gamma / \mathrm{H} \beta$ ratio of 0.466 (Osterbrock 1989). Since the stellar absorptions underlying Balmer emission-lines have been corrected carefully by using the synthesized SSP, the dust extinction

\footnotetext{
2 http://www.starlight.ufsc.br

${ }^{3}$ IRAF is distributed by the National Optical Astronomical Observatories, which is operated by the Association of Universities for Research in Astronomy, Inc., under cooperative agreement with the National Science Foundation.
} 

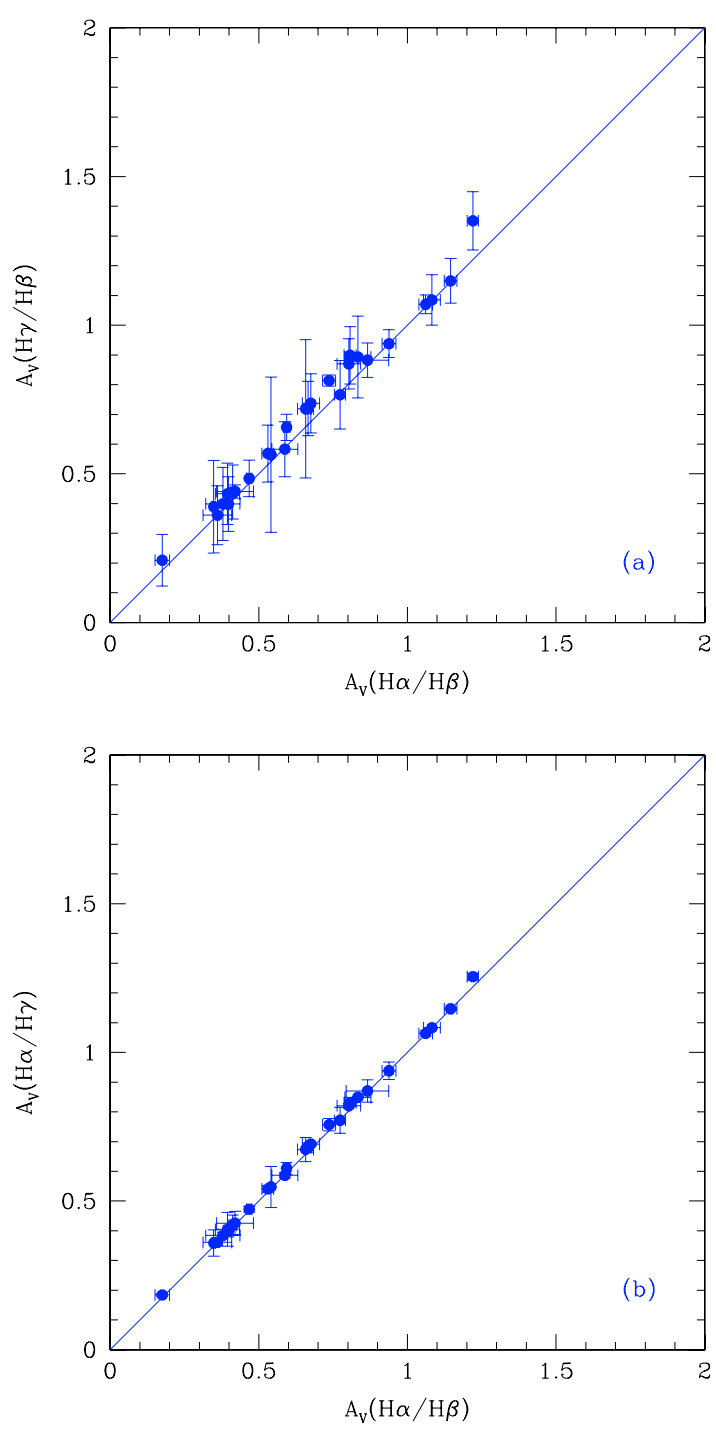

Fig. 3. Comparisons between the extinction values derived from $\mathrm{H} \alpha / \mathrm{H} \beta$, $\mathrm{H} \gamma / \mathrm{H} \beta$ and $\mathrm{H} \alpha / \mathrm{H} \gamma$ for the sample galaxies. $A_{V}(\mathrm{H} \alpha / \mathrm{H} \beta)$ is adopted as the actual extinction in this study.

values derived from the three line ratios should be almost identical. Figure 3 shows the excellent consistency of the derived values for the dust extinction.

The uncertainties of the flux measurements are dominated by the underlying stellar absorption correction. Another source comes from the independent measurements by two of us, YCL and SYY. Figure 3 shows that the error-bars of $A_{V}(\mathrm{H} \gamma / \mathrm{H} \beta)$ are moderately larger than the other two from $\mathrm{H} \alpha / \mathrm{H} \beta$ and $\mathrm{H} \alpha / \mathrm{H} \gamma$, simply because the $\mathrm{H} \gamma$ line is weaker than the other two lines. $\mathrm{H} \alpha$ is the strongest of these three lines, and is affected less than the other two by the stellar absorption. In the following, we adopt $A_{V}(\mathrm{H} \alpha / \mathrm{H} \beta)$ (given in Table 2) as the actual extinction inside the galaxies.

\section{Consistency checks of the empirical $\mathrm{O} / \mathrm{H}$ estimates using the $R_{23}$ method}

It is necessary to re-derive the relationship between $(\mathrm{O} / \mathrm{H})$ abundances and stellar masses and $R_{23}$ parameters for nearby starforming galaxies on the basis of the SDSS-DR4 database by following the method of T04 since our sample galaxies are selected from DR4. This revises somewhat the calibrations obtained from the DR2 database by T04, but should be more reasonable for this study.

Figures 4a,b show the relationship between $12+\log (\mathrm{O} / \mathrm{H})$ and $\log \left(M_{*}\right)$ and $\log \left(R_{23}\right)$ for the DR4 star-forming galaxies ( $\sim 85000$, the small points). The dashed lines are the calibrations of T04 obtained from DR2, and the solid lines are the re-derived relations from DR4, given as the following formulas:

$$
\begin{aligned}
12+\log (\mathrm{O} / \mathrm{H})= & -5.292+2.568\left(\log M_{*}\right)-0.11441\left(\log M_{*}\right)^{2},(1) \\
12+\log (\mathrm{O} / \mathrm{H})= & 9.412-2.251\left(\log R_{23}\right)+4.885\left(\log R_{23}\right)^{2} \\
& -4.564\left(\log R_{23}\right)^{3} .
\end{aligned}
$$

They are obtained by fitting the median values in the bins of $0.1 \mathrm{dex}$ in $\log \left(M_{*}\right)$ and $0.05 \mathrm{dex}$ in $\log (\mathrm{O} / \mathrm{H})$, respectively.

Figure $4 \mathrm{a}, \mathrm{b}$ reveals the differences between the results from DR4 and DR2 for these two relationships which might be due to the expansion of the database. Equation (1) will result in a lower $\log (\mathrm{O} / \mathrm{H})$ abundance than the previous calibration at the given stellar mass, generally about 0.1 dex. Equation (2) will result in a higher and $\operatorname{lower} \log (\mathrm{O} / \mathrm{H})$ abundance than the previous one at the given $\log \left(R_{23}\right)$ at the metal-rich and metal-poor ends, respectively.

Figure $4 \mathrm{c}$ shows our selected galaxies (the small blue points) that we extracted from the whole sample of SDSS-DR4 galaxies following the criteria described in Sect. 2. The large solid circles refer to the median-value points in the 27 mass bins given in Table 1. The solid line is the same as in Fig. 4a and Eq. (1). It shows that these median-value points of the selected samples are very close to the median-value relation of the whole sample (the solid line). Notice however that it slightly biases $\mathrm{O} / \mathrm{H}$ values towards lower metallicities at the highest mass end.

Figure 5 presents the $12+\log (\mathrm{O} / \mathrm{H})_{R_{23}}$ versus $\log \left(M_{*}\right)$ relationship of our 27 stacked spectra. Their $12+\log (\mathrm{O} / \mathrm{H})_{R_{23}}$ abundances are estimated from the extinction-corrected $R_{23}$ parameters following Eq. (2). The corresponding stellar mass in each bin is the middle value there (see Table 1). Notice that the data points obtained from the stacked spectra are very similar to the large filled circles in Fig. 4c, i.e., the actual median-value points of the sample galaxies in the same mass bins. Moreover, the stacked spectra provide values quite close to the solid line, which represents the median-value relation of the whole sample. This implies that for the empirical $\mathrm{O} / \mathrm{H}$ estimate using the $R_{23}$ method, our stacking method provides a good representation of the median value for the whole sample, and that the stacking method is able to give a reasonable estimate of the behavior of the global mass-metallicity relationship.

\section{The "direct" oxygen abundances derived from electron temperature $T_{e}$}

A two-zone model for the temperature structure within the galactic gas was adopted. In this model, $T_{\mathrm{e}}\left(\left[\begin{array}{ll}\mathrm{O} & \mathrm{II}\end{array}\right]\right.$ ) (in $\mathrm{K}$, as $t_{2}$ in $10^{4} \mathrm{~K}$ ) represents the temperature for low-ionization species such as $\mathrm{O}^{+}\left(\right.$and $\left.\mathrm{N}^{+}\right)$, while $T_{\mathrm{e}}([\mathrm{O} \mathrm{III}])$ (in $\mathrm{K}$, as $t_{3}$ in $10^{4} \mathrm{~K}$ ) represents the temperature for high-ionization species such as $\mathrm{O}^{++}$.

We estimate $t_{2}$ from the ratio of [O $\left[\begin{array}{ll}\mathrm{O}\end{array}\right] \lambda \lambda 7320,7330$ to $\left[\begin{array}{ll}\mathrm{O} & \mathrm{II}\end{array}\right] \lambda 3727$ by using the task temden.nebular in the IRAF/STSDAS package (de Robertis et al. 1987; Shaw \& Dufour 1995); $t_{3}$ in the high-ionization zone is determined from $t_{2}$ following an equation derived by fitting $\mathrm{HII}$ region models. Several versions of the $t_{2}-t_{3}$ relationship have been proposed 

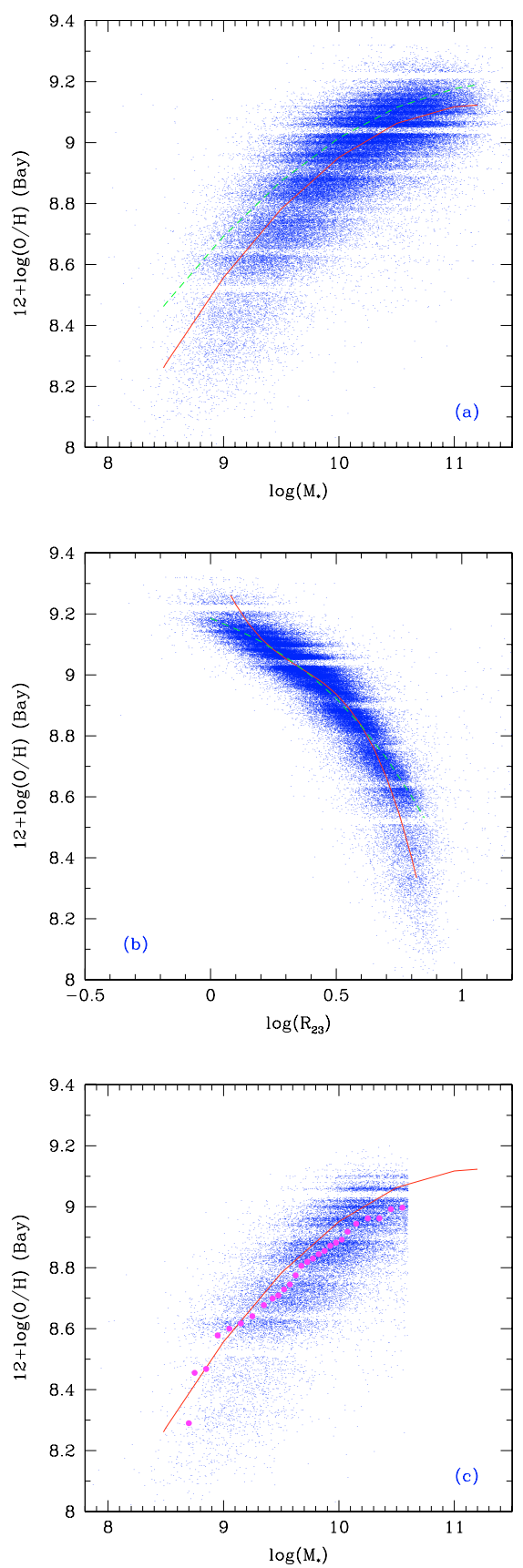

Fig. 4. Relation of the $\log (\mathrm{O} / \mathrm{H})$ abundances versus the stellar masses a) and versus the $\log \left(R_{23}\right)$ b) of the nearby star-forming galaxies selected from SDSS-DR4 (the small points, $~ 85000$ ). The two solid lines in a) and b) refer to the new calibrations derived from the SDSSDR4 star-forming galaxies with $8.5 \leq \log \left(M_{*}\right) \leq 11.3$ (Eq. (1)), and $8.3 \leq 12+\log (\mathrm{O} / \mathrm{H}) \leq 9.2$ (Eq. (2)), respectively. The dashed lines refer to the calibrations obtained by T04 from DR2 database. c) represents the sample of galaxies selected for this study. The large filled circles refer to the median-value points in the mass bins following Table 1, which are very close to the solid line, the same one as in Fig. 4a. (Please see the online color version of the plots for the lines.)

(Pilyugin et al. 2006). We use the one given by Garnett (1992), which is valid over the range $2000 \mathrm{~K}<T_{\mathrm{e}}([\mathrm{O}$ III] $)<18000 \mathrm{~K}$, and has been widely used:

$t_{2}=0.7 t_{3}+0.3$.

We can then obtain the "direct" oxygen abundances from electron temperature for these combined galaxies by using the

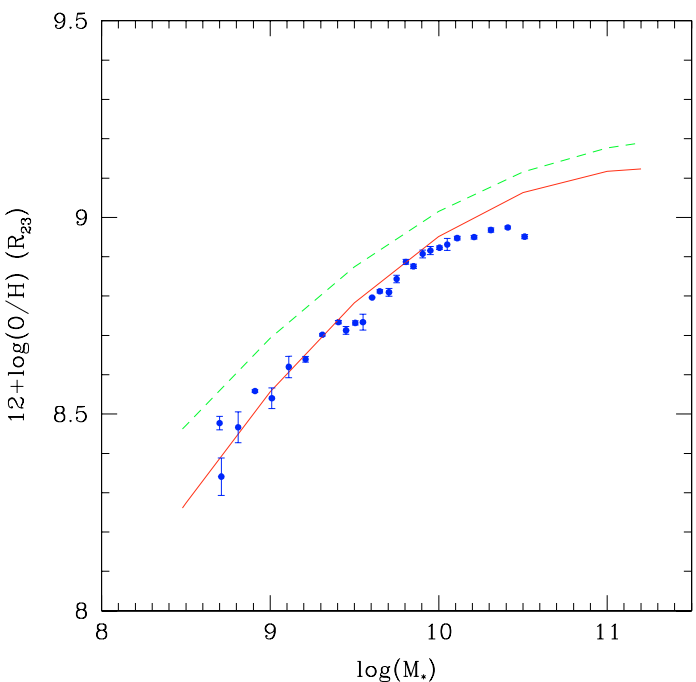

Fig. 5. Relationship between the $12+\log (\mathrm{O} / \mathrm{H})_{R_{23}}$ abundances and the stellar masses for our 27 combined galaxies (the points). The solid and dashed lines are the same as in Fig. 4a.

equations published by Izotov et al. (2006) for the determination of the oxygen abundances in H II regions for a five-level atom. They used the atomic data from the references listed in Stasińska (2005). The formulas are

$$
\begin{aligned}
12+\log \left(\mathrm{O}^{+} / \mathrm{H}^{+}\right)= & \log \left(I_{[\mathrm{OII}] \lambda 3726+\lambda 3729} / I_{\mathrm{H} \beta}\right) \\
& +5.961+\frac{1.676}{t_{2}}-0.40 \log t_{2}-0.034 t_{2} \\
& +\log \left(1+1.35 x_{2}\right),
\end{aligned}
$$

where $x_{2}=10^{-4} n_{\mathrm{e}} t_{2}^{-1 / 2}$, and $n_{\mathrm{e}}$ is the electron density in $\mathrm{cm}^{-3}$, and

$$
\begin{aligned}
& 12+\log \left(\mathrm{O}^{++} / \mathrm{H}^{+}\right)=\log \left(I_{[\mathrm{OIII}] \lambda 4959+\lambda 5007} / I_{\mathrm{H} \beta}\right) \\
& +6.200+\frac{1.251}{t_{3}}-0.55 \log t_{3}-0.014 t_{3} \text {. }
\end{aligned}
$$

The total oxygen abundances are derived from the following equation:

$\frac{\mathrm{O}}{\mathrm{H}}=\frac{\mathrm{O}^{+}}{\mathrm{H}^{+}}+\frac{\mathrm{O}^{++}}{\mathrm{H}^{+}}$.

The electron densities in the ionized gas of the galaxies are calculated from the line ratios [S II] $26717 /[\mathrm{S}$ II] $\lambda 6731$ (Cols. 2, 3 in Table 2) by using the five-level statistical equilibrium model in the task temden.nebular in the IRAF/STSDAS package at $T_{\mathrm{e}}=$ $10000 \mathrm{~K}$. Notice that $x_{2}$ in Eq. (4) has a very small impact since it is generally less than 0.1 with $n_{\mathrm{e}}<10^{3} \mathrm{~cm}^{-3}$.

Table 2 lists the derived properties of the combined galaxies, including the mass-bin, the [S II] $] 6717 /[\mathrm{S}$ II] $] 6731$ ratios, the electron density, the $[\mathrm{O}$ II $] \lambda \lambda 7320,7330 /[\mathrm{O}$ II $] \lambda 3727$ ratios, the electron temperature $T_{\mathrm{e}}([\mathrm{O} \mathrm{II}])$ (in $\left.\mathrm{K}\right), T_{\mathrm{e}}([\mathrm{O} \mathrm{III}])$ (in $\mathrm{K}$ ), and the $T_{\mathrm{e}}$-based $12+\log (\mathrm{O} / \mathrm{H})$ abundances, which are approximately 8.0 to 9.0 .

Figure 6 shows the relationship between the $T_{\mathrm{e}}$-based $\log (\mathrm{O} / \mathrm{H})$ abundances and the stellar masses $\log \left(M_{*}\right)$ for the combined galaxies (the filled circles). The thick long-dashed line is the linear least-square fit for the data point and is given by

$12+\log (\mathrm{O} / \mathrm{H})=6.223+0.231 \times\left(\log M_{*}\right)$,

with an rms about $0.12 \mathrm{dex}$, which shows the increasing $\log (\mathrm{O} / \mathrm{H})$ following the stellar masses. 


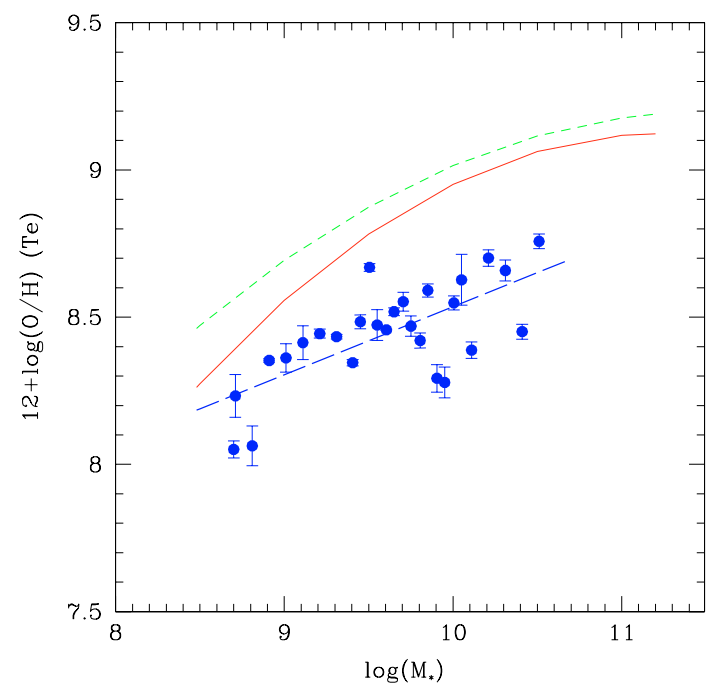

Fig. 6. Relationship between the $(\mathrm{O} / \mathrm{H})$ abundances derived from $T_{\mathrm{e}}$ and the stellar masses of the combined galaxies. The long-dashed line is the linear least-square fit for the data points. The solid and short-dashed lines are the same as in Fig. 5.

This shows that the $T_{\mathrm{e}}$-based $\log (\mathrm{O} / \mathrm{H})$ abundances of the upper branch are generally about $0.2-0.4 \mathrm{dex}$ (down to $0.6 \mathrm{dex}$ ) lower than the $R_{23}$-based abundances (the solid and dashed lines, the same as in Fig. 5) at a given stellar mass. The error-bars marked here come from the flux measurements. We did not consider the uncertainties from the conversion relationship between $t_{2}$ and $t_{3}$, and the temperature fluctuations and gradients (see the discussions in Sect. 8).

\section{Comparisons between the $T_{\mathrm{e}}$-based $(\mathrm{O} / \mathrm{H})$ and those from empirical strong-line methods}

In this section, we compare the $T_{\mathrm{e}}$-based $\mathrm{O} / \mathrm{H}$ abundances of the sample galaxies with those derived from other "strong-line" ratios, such as $R_{23}, P$-method and $N 2(=\log ([\mathrm{N} \mathrm{II}] / \mathrm{H} \alpha))$ index. The corresponding parameters and the derived $(\mathrm{O} / \mathrm{H})$ abundances are presented in Table 2.

\subsection{The $R_{23}$ method}

The $R_{23}$ parameter is widely used to estimate metallicities for metal-rich galaxies. The empirical relationship between $\mathrm{O} / \mathrm{H}$ and $R_{23}$ has been suggested and extensively discussed in the literature (see Pagel et al. 1979; McGaugh 1991; Kobulnicky et al. 1999; Tremonti et al. 2004, and references therein; Sect. 4 in this study). However, some recent observations show that the $R_{23}$ will overestimate the $\log (\mathrm{O} / \mathrm{H})$ abundances by $0.2-0.5 \mathrm{dex}$ on the basis of about a hundred $\mathrm{H}$ II regions in the spiral galaxies M101 and M51 (KBG03; Bresolin et al. 2004, 2005; Bresolin 2007; Garnett et al. 2004a).

Figure 7a shows how these combined SDSS galaxies (the filled circles) are distributed in the relationship between $\log \left(R_{23}\right)$ and $12+\log (\mathrm{O} / \mathrm{H})_{T_{\mathrm{e}}}$. The empirical $R_{23}$ calibrations obtained by T04 (the dot-dashed line), K99 (the short-dashed lines), Kobulnicky \& Kewley (2004, KK04; the dotted line) and that derived from the SDSS-DR4 database in this study (the longdashed line, see Sect. 4) are also given. The H II regions studied by KBG03 and Castellanos et al. (2002, hereafter CDT02), and metal-poor galaxies studied by Yin et al. (2007a) are also plotted. We will not present here other $\mathrm{H}$ II regions from
Garnett et al. (2004a) and Bresolin (2007) since these are special H II regions in the center of the spiral galaxies and are not exactly the same case as galaxies with their integrated light of many H II regions studied here.

The combination of all these observational data with $T_{\mathrm{e}^{-}}$ based $(\mathrm{O} / \mathrm{H})$ estimates shows that the $R_{23}$ parameters result in double-valued abundances with a transition region of 7.9-8.2 of $12+\log (\mathrm{O} / \mathrm{H})$ and $0.9-1.0$ of $\log \left(R_{23}\right)$. We consistently find that most of our sample galaxies lie in the upper-branch with their $8.3<12+\log (\mathrm{O} / \mathrm{H})<9.0$ and $0.4<\log \left(R_{23}\right)<0.75$. Our samples are similar to those of CDT02, and more metal-rich than those of KBG03 and Yin et al. (2007a). Moreover, it is clear that generally the empirical calibrations for upper-branch result in higher $\log (\mathrm{O} / \mathrm{H})$ abundances at a given $R_{23}$, up to 0.6 dex.

We obtain a third-order polynomial fit for the total sample of these observational data (702, without considering the two most metal-rich $\mathrm{H}$ II regions from CDT02 and the three scattered ones with $\log \left(R_{23}\right)>1.0$ from the SDSS) for $\log \left(R_{23}\right)$ vs. $\log (\mathrm{O} / \mathrm{H})$, which is given by the solid line in Fig. $7 \mathrm{~b}$, and by

$\log R_{23}=73.13-32.78 x+4.806 x^{2}-0.230 x^{3}$,

where $x=12+\log (\mathrm{O} / \mathrm{H})$. It reveals the discrepancy between this newly derived calibration based on the $T_{\mathrm{e}}-(\mathrm{O} / \mathrm{H})$ abundances and those derived from the previous strong-line empirical calibrations (other lines).

To show this discrepancy more clearly and directly, we compare the $(\mathrm{O} / \mathrm{H})_{R_{23}}$ and $(\mathrm{O} / \mathrm{H})_{T_{\mathrm{e}}}$ in Fig. 8. In Fig. 8a, the $(\mathrm{O} / \mathrm{H})_{R_{23}}$ abundances of all the data points are estimated by using Eq. (2), which is the new calibration derived from the DR4 database in this study. In Fig. $8 \mathrm{~b}$, the $(\mathrm{O} / \mathrm{H})_{R_{23}}$ abundances are estimated from T04's calibration derived from DR2, which could be representative of other empirical calibrations. They show that the $R_{23}$ parameter will overestimate the actual $\mathrm{O} / \mathrm{H}$ abundances by a factor up to 0.6 dex for the moderate metal-rich galaxies. For the objects within the transition region of abundances, the calibrations of $R_{23}$ derived from DR4 will give consistent abundances as $T_{\mathrm{e}}$, but others (e.g. DR2) will still overestimate the $\mathrm{O} / \mathrm{H}$ abundances (see the open circles in Figs. 8a,b).

\subsection{The P-method}

It is worth while comparing the $T_{\mathrm{e}}$-based abundances with those derived from the $P$-method, which is often assumed to give "reliable" metallicities for the metal-rich galaxies (Liang \& Yin 2007). Pilyugin (2000, 2001a,b; as P01) suggested his $P$-method to estimate the oxygen abundances of galaxies in which the oxygen abundance can be derived from two parameters, $R_{23}$ and $P(=[\mathrm{O}$ III $] 4959,5007 /([\mathrm{O}$ II $] 3727+[\mathrm{O}$ III $] 4959,5007))$. He derived the $\mathrm{O} / \mathrm{H}=f\left(R_{23}, P\right)$ formulas from a sample of metalpoor $\mathrm{H}$ II regions with $7.1<12+\log (\mathrm{O} / \mathrm{H})_{T_{\mathrm{e}}}<7.95$ and a sample of moderately metal-rich $\mathrm{H}$ II regions with $8.2<$ $12+\log (\mathrm{O} / \mathrm{H})_{T_{\mathrm{e}}}<8.7$. The fitting relation for moderately metalrich $\mathrm{H}$ II regions ( 40) is given as Eq. (8) in Pilyugin (2001a). Pilyugin \& Thuran (2005, as PT05) have renewed the $P$ calibrations by including several improvements, such as enlarging the sample ( $\sim 104$ metal-rich H II regions). However, on the basis of $\sim 20000$ metal-rich star-forming galaxies from the SDSS-DR2, we found that the oxygen abundances derived from this revised $P$-method (PT05) are $\sim 0.19$ dex lower than those derived from the previous one $(\mathrm{P} 01)$, and in particular are $\sim 0.60 \mathrm{dex}$ lower than the Bayesian abundances obtained by the MPA/JHU group (Yin et al. 2007b). These abundance discrepancies strongly correlate with the $P$ parameter and weakly depend on the $\log \left(R_{23}\right)$ 

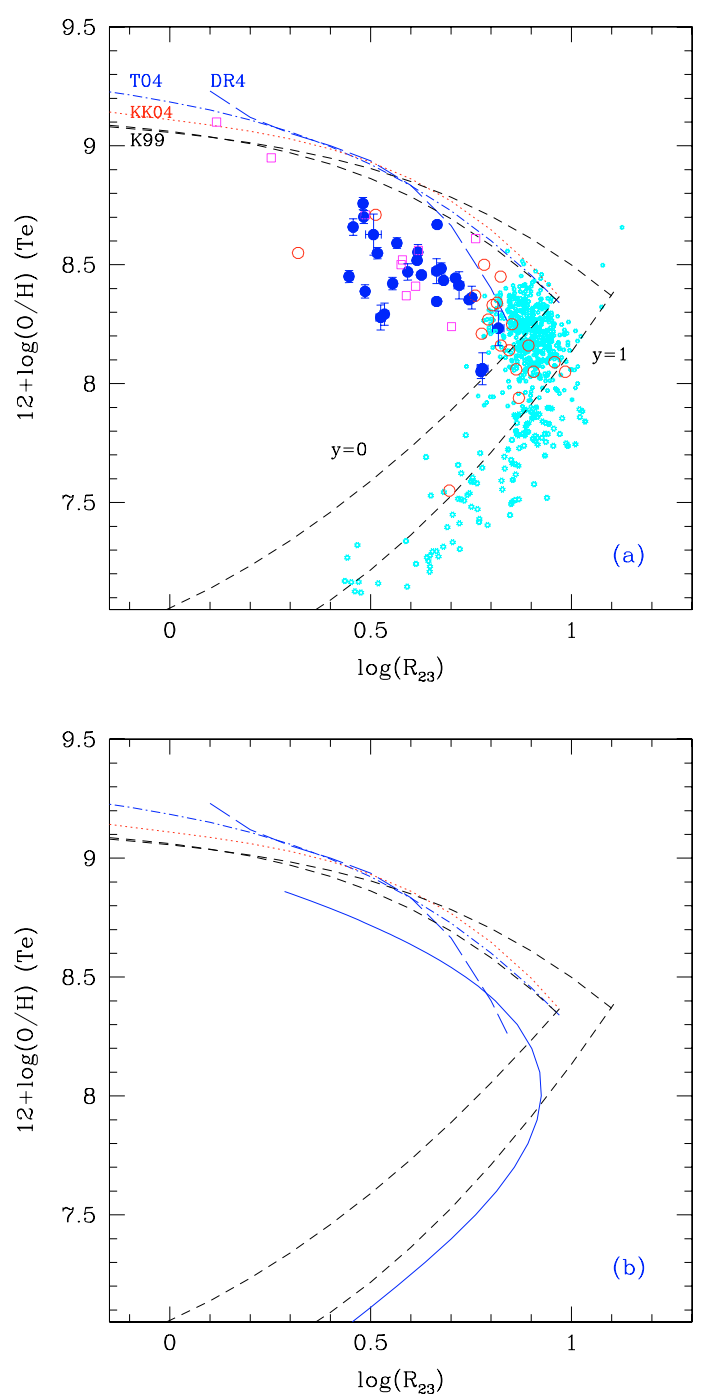

Fig. 7. a) The relation of the $T_{\mathrm{e}}$-based $(\mathrm{O} / \mathrm{H})$ abundances with $R_{23}$ parameters for our sample galaxies (the filled circles). Correlation with those from the literature: the large open circles refer to the $\mathrm{H}$ II regions in M101 taken from KBG03, the squares represent the H II regions given by $\mathrm{CDT} 02$, and the small open circles refer the lowmetallicity H II regions and galaxies taken from Yin et al. (2007a). Some empirical calibrations are also given: those of T04 (the dotdashed line), Kobulnicky et al. (1999; K99, the two dashed-lines, $y=$ $\log O_{32}=\log ([\mathrm{O}$ III $] 4959,5007 /[\mathrm{O}$ II $\left.] 3727)\right)$, Kobulnicky \& Kewley (2004, KK04, the dotted line), and that derived from SDSS-DR4 in this study (DR4, the long-dashed line). b) The third-order polynomial fit (the solid line) for all the observational data points given in a), also given as Eq. (8). Other calibrations from the literature are the same as in a).

parameter, which is especially serious for the case of $P<0.55$ (Yin et al. 2007b; PT05; P01).

In Fig. 9, we compare the $T_{\mathrm{e}}$-based $\log (\mathrm{O} / \mathrm{H})$ abundances of the 27 combined galaxies with those derived by using the $P$-method of P01 for the metal-rich branch. The H II regions studied by KBG03 and CDT02 are also plotted. It seems that the $P$-method will almost result in a constant $(\mathrm{O} / \mathrm{H})$ abundance of about $12+\log (\mathrm{O} / \mathrm{H}) \sim 8.4$ for both of our samples and most of the data from the literature, although the $\log (\mathrm{O} / \mathrm{H})_{P}$ and $\log (\mathrm{O} / \mathrm{H})_{T \mathrm{e}}$ are consistent within $0.3 \mathrm{dex}$. The reason for our sample galaxies may be that the P01's $P$-method is not quite reliable to derive their oxygen abundances. This is because all our
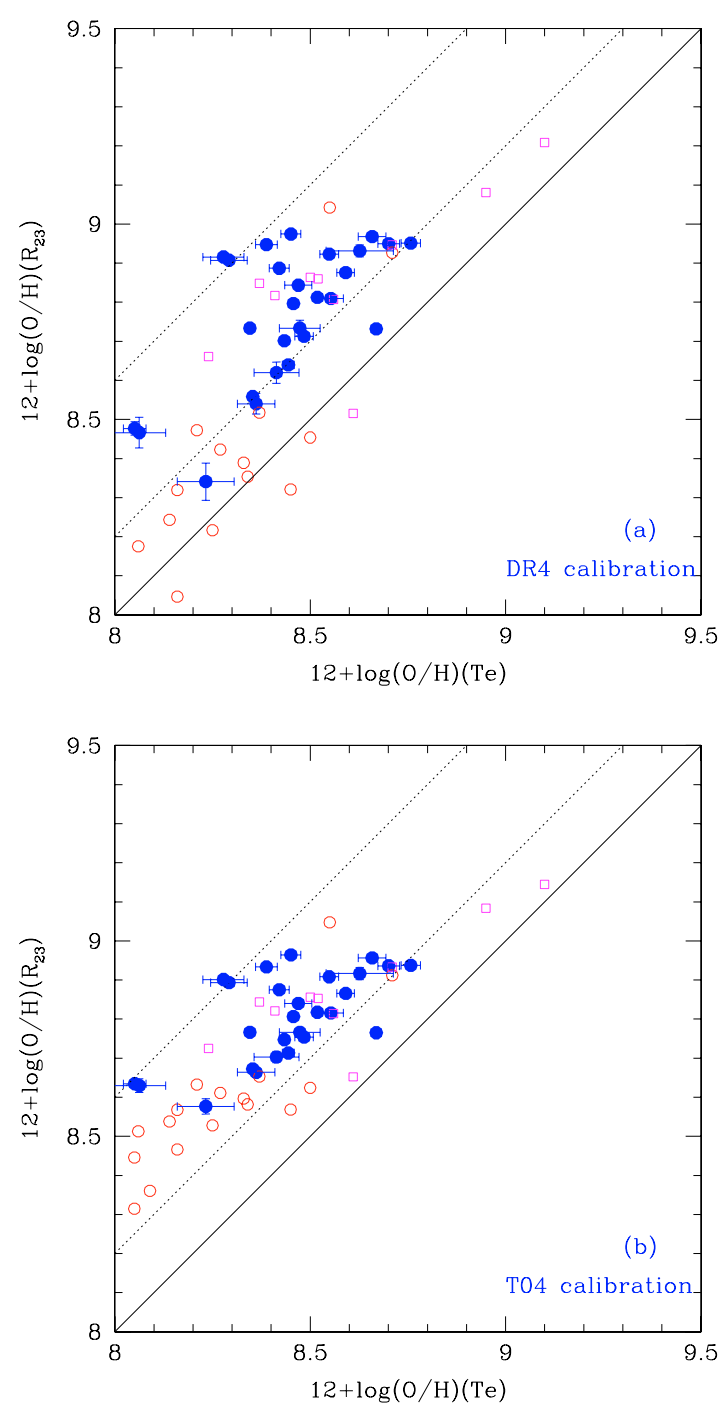

Fig. 8. Comparison between the $T_{\mathrm{e}}$-based and the $R_{23}$-based $\log (\mathrm{O} / \mathrm{H})$ abundances of the sample galaxies: a) the $R_{23}$-calibration was derived from DR4 with $12+\log (\mathrm{O} / \mathrm{H})>8.3$ in this study (Eq. (2)); and b) the $R_{23}$-calibration was derived from DR2 by T04 with $12+\log (\mathrm{O} / \mathrm{H})>8.5$. The symbols as the same as in Fig. 7. The solid line in each panel represents the equal relation, and the two dashed lines are the relations by increasing $12+\log (\mathrm{O} / \mathrm{H})_{R_{23}}$ abundances by 0.2 and 0.6 dex, respectively.

moderate metal-rich sample galaxies selected from the SDSS have $P<0.55$ (see Table 2), due to the [O III] line becoming weaker in the metal-rich environments, which is the case for most of the metal-rich SDSS galaxies (see Yin et al. 2007b). But the calibration formula of P01 (as well PT05) was derived from fitting the sample $\mathrm{H}$ II regions that mostly have $P>0.55$, and only a few of their samples have $P<0.55$. The samples of CDT02 have similar $P$-values to ours, and show similar trends to those described here. The samples of KBG03 generally have higher $P$ values than 0.55 . The reason for their discrepancy from the equal-line may be that they are just in the turn-over region of abundances when strong-line calibration cannot give accurate abundances.

\subsection{The N2 method}

The $[\mathrm{N}$ II $] / \mathrm{H} \alpha$ emission-line ratio (as $N 2=\log ([\mathrm{N}$ II $] \lambda 6583 /$ $\mathrm{H} \alpha)$ ) is useful to estimate the metallicities of galaxies 


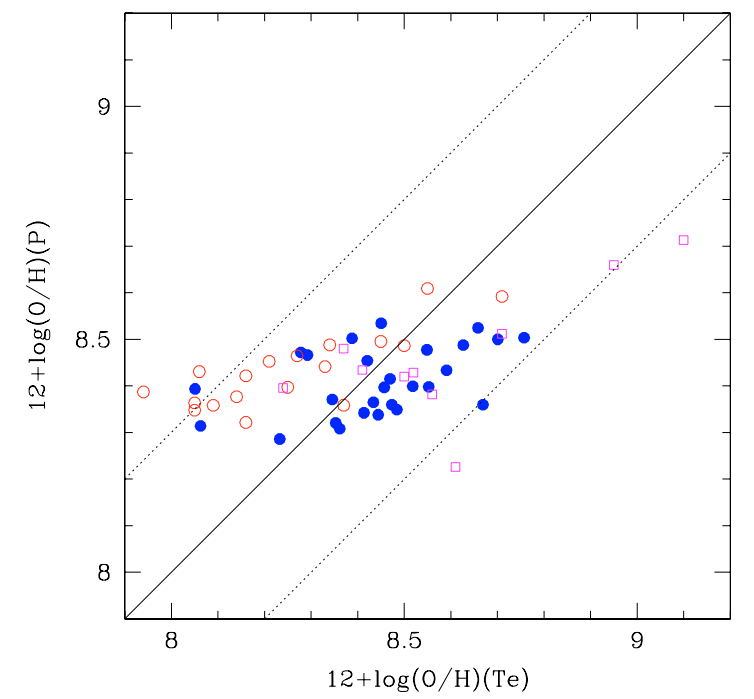

Fig. 9. Comparison between the $T_{\mathrm{e}}$-based $(\mathrm{O} / \mathrm{H})$ abundances and those estimated from $P$-method for our sample galaxies. The symbols as the same as in Fig. 7.

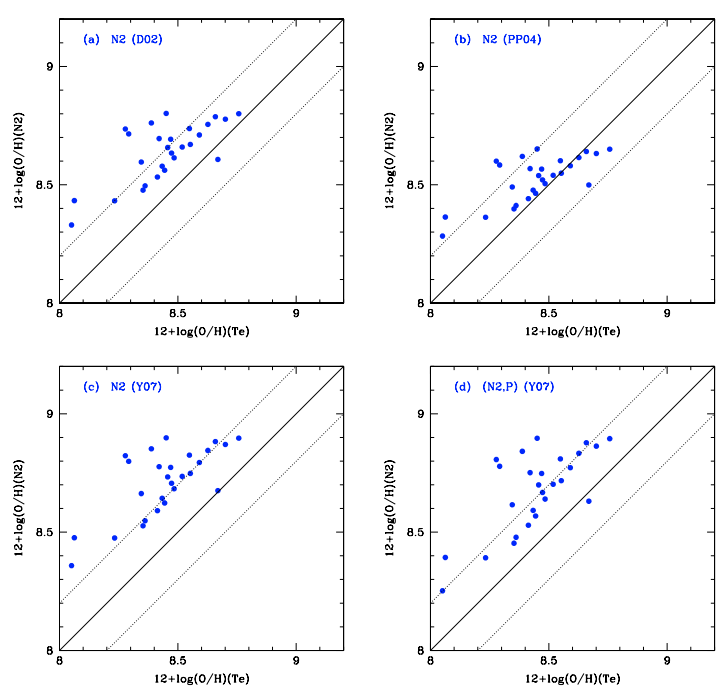

Fig. 10. Comparisons between the $T_{\mathrm{e}}$-based $(\mathrm{O} / \mathrm{H})$ abundances of the sample galaxies with those estimated from $N 2$ index calibrations: a) from $\mathrm{D} 02 ; \mathbf{b})$ from $\mathrm{PP} 04 ; \mathbf{c})$ from $\mathrm{Y} 07 ; \mathbf{d})$ the $(N 2, P)$ calibration from Y07.

(Liang et al. 2006; Yin et al. 2007a, as Y07; Pettini \& Pagel 2004, as PP04; Denicoló et al. 2002, as D02; Kewley \& Dopita 2002), though it may depend on the N-enrichment history of the galaxies (Liang \& Yin 2007), and ionization parameters (Kewley $\&$ Dopita 2002). $N 2$ is not greatly affected by dust extinction due to the close wavelength positions of [N II] and $\mathrm{H} \alpha$, and the developed near infrared spectroscopic instruments can gather these two lines for the galaxies with intermediate and high redshifts, which stand in the early epoch of the universe.

Here we compare these $T_{\mathrm{e}}$-based $(\mathrm{O} / \mathrm{H})$ abundances with those derived from the $N 2$ calibrations of D02, $\mathrm{PP} 04, \mathrm{Y} 07$, also the $(N 2, P)$ calibration from Y07 $(P=[\mathrm{O}$ III $] /([\mathrm{O}$ II $]+[\mathrm{O}$ III $]))$, given in Figs. 10a-d. They show that the $N 2$-calibration given by PP04 could give $(\mathrm{O} / \mathrm{H})$ abundances that are more consistent with the $T_{\mathrm{e}}$-based ones, while other $N 2$-calibrations often overestimate the $(\mathrm{O} / \mathrm{H})$ abundances for these moderate metal-rich galaxies.

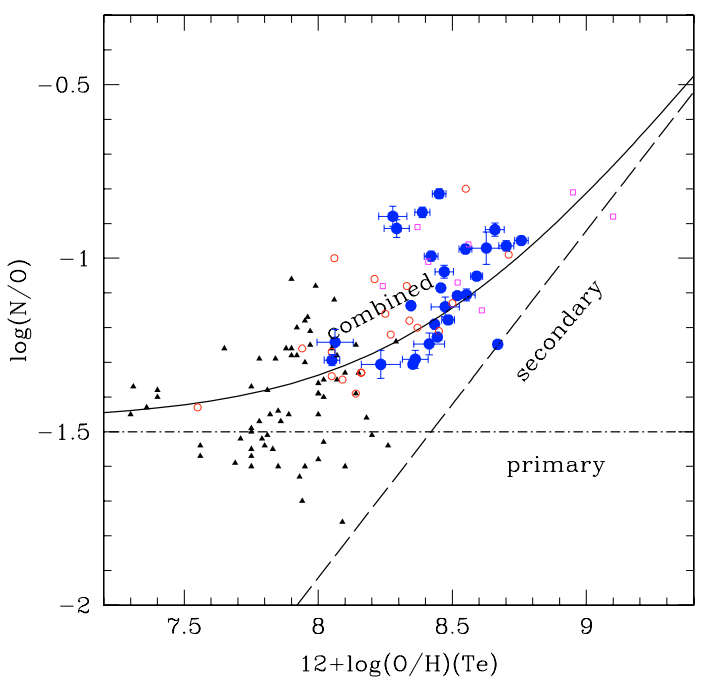

Fig. 11. $\log (\mathrm{N} / \mathrm{O})$ abundance ratios of the sample galaxies as functions of their $12+\log (\mathrm{O} / \mathrm{H})$ abundances derived from $T_{\mathrm{e}}$ (the large filled circles). The dot-dashed line refers to the "primary" component of nitrogen, the long-dashed line refers to the "secondary" component of nitrogen, and the solid line refers to the combination of these two components, which are taken from Vila-Costas \& Edmunds (1993). Other recent observations are also given: the triangles represent the dwarf galaxies studied by van Zee \& Haynes (2006), and the open circles and squares represent the extragalactic $\mathrm{H}$ II regions studied by KBG03 and CDT02, respectively.

\section{The $\log (\mathrm{N} / \mathrm{O})$ abundance ratios}

The "direct" electron temperature in the low-ionization region of the galactic gas and the high quality optical spectra make it possible to estimate the $\log (\mathrm{N} / \mathrm{O})$ abundances of these combined galaxies properly, which is useful to understand the "primary" and/or "secondary" origin of the nitrogen element.

If the "seed" oxygen and carbon for the production of nitrogen via the $\mathrm{CNO}$ processing are those incorporated into a star at its formation and a constant mass fraction is processed, then the amount of nitrogen produced is proportional to the initial heavyelement abundance, and the nitrogen synthesis is said to be "secondary". If the oxygen and carbon are produced in the star prior to the CNO cycling (e.g. by helium burning in a core, followed by $\mathrm{CNO}$ cycling of this material mixed into a hydrogen-burning shell), then the amount of nitrogen produced may be fairly independent of the initial heavy-element abundance of the star, and the synthesis is said to be "primary" (Vila-Costas \& Edmunds 1993). In general, primary nitrogen production is independent of metallicity, while secondary production is a linear function of it.

We adopt the low-ionization region temperature $t_{2}$ for $\left[\begin{array}{ll}\mathrm{N} & \mathrm{II}\end{array}\right]$ emission regions $\left(t_{2}\left(\left[\begin{array}{ll}\mathrm{N} & \mathrm{II}\end{array}\right)=t_{2}\left(\left[\begin{array}{ll}\mathrm{O} & \mathrm{II}\end{array}\right]\right)\right)\right.$ and the [N II] $\lambda \lambda 6548,6583 /[\mathrm{O}$ II] $] \lambda 3727$ ratio to derive the $\log (\mathrm{N} / \mathrm{O})$ abundances of these combined galaxies by using the formulas of Izotov et al. (2006) for the $\log \left(\frac{\mathrm{N}^{+}}{\mathrm{H}^{+}}\right)$and $\log \left(\frac{\mathrm{O}^{+}}{\mathrm{H}^{+}}\right)$abundances, with $\frac{\mathrm{N}}{\mathrm{O}}=\frac{\mathrm{N}^{+}}{\mathrm{O}^{+}}$. Figure 11 (Table 2 as well) presents the results. These $T_{\mathrm{e}}$-based $(\mathrm{O} / \mathrm{H})$ and $(\mathrm{N} / \mathrm{O})$ abundances show that the sources of nitrogen for these moderate metal-rich galaxies are consistent with the combination of the primary and secondary components. This is a robust investigation on the production of nitrogen for these moderate metal-rich galaxies since both the $\mathrm{N}$ and $\mathrm{O}$ abundances are obtained from electron temperature on the basis of their integrated light. The H II regions of KBG03 and CDT02 also show a similar nitrogen source to our samples. Most of the 


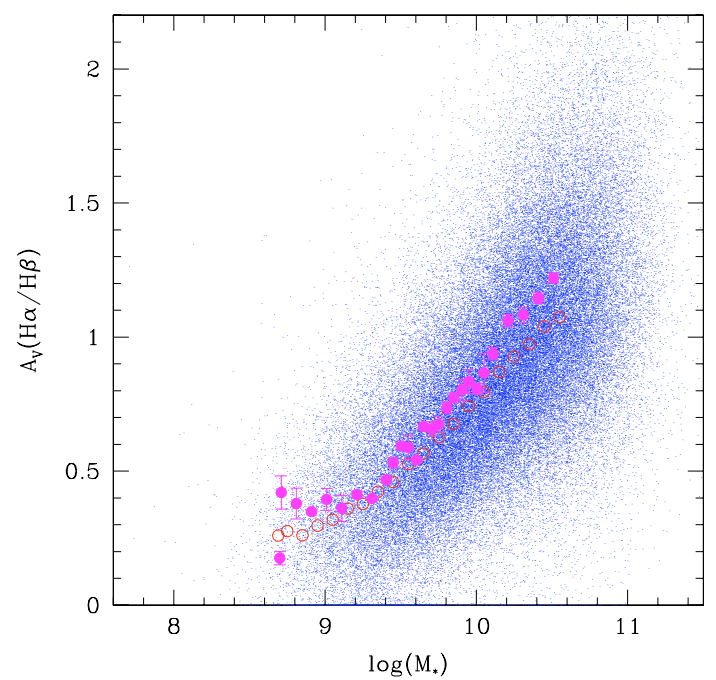

Fig. 12. Relationship between dust extinction and stellar masses of the sample galaxies. The large filled circles refer to the results from the stacked spectra of the galaxies in the 27 mass bins. The small points refer to the star-forming galaxies from SDSS DR4 (the same as in Fig. 4a), and the open circles refer to the median values of them. (Please see the online color version of the plot for the points, especially for the open circles.)

dwarf galaxies taken from van Zee \& Haynes (2006) show the primary source of their nitrogen.

\section{Discussion}

Our procedure to derive $T_{\mathrm{e}}$-based oxygen abundances for the metal-rich galaxies may be affected by some biases that need to be discussed further. The major contributors for such biases are the dust estimates, the stacking method, and the uncertainties related to the temperature determination.

\subsection{Dust extinction}

Our method is strongly dependent on the dust extinction, since the determination of the temperature depends on the ratio of red over blue emission lines. However, the $\mathrm{S} / \mathrm{N}$ of the stacked spectra is so large that our estimate of $A_{V}$ is very accurate. On the other hand, one may wonder whether by stacking spectra, we still present the representative of the mean properties of individual galaxies.

We confirm the strong correlation between the dust extinction $\left(A_{V}\right)$ and the stellar masses of star-forming galaxies, which, as expected, means the more massive galaxies generally have more heavy dust attenuation (see also Salim et al. 2007). In Fig. 12 the large filled circles represent our stacked galaxies, the small points represent the whole sample of the star-forming galaxies from SDSS DR4 (same as in Fig. 4a), and the large open circles represent the median values of this whole sample in the corresponding mass bins. In most of the considered stellar mass range, the stacked spectra provide a $A_{V}-M_{*}$ relationship almost identical to that of the whole sample, meaning that the stacking method and selection criteria do not cause significant biases in the estimation of the dust extinction. Notice however that at the highest mass range, stacking spectra provide a slightly higher extinction, which may well be due to the selection method. Indeed, by selecting stronger star forming galaxies than the average, it is not surprising to find slightly higher dust extinction.

\subsection{Are stacked spectra providing a good representation of real galaxies?}

The "stacking" method has been used widely in astronomical study, e.g., Zheng et al. (2006) and Chang et al. (2006), who stacked the images or property parameters of galaxies to study their properties. There are also examples of uses of stacked spectra: Mathis et al. (2006) stacked the spectra in several mass bins to study the cosmic star formation history; Baldry et al. (2002) analyzed the stacked low-resolution spectra of 166000 galaxies at redshift $0.03<z<0.25$ extracted from the 2dFGS; Schiavon et al. (2006) used the stacked Keck DEIMOS spectra to study the ages and metallicities of some red galaxies; and Wild et al. (2007) used the stacked SDSS spectra in redshift bins to study the [O II] $\lambda 3727$ nebular emission from the galaxies hosting $\mathrm{Ca}$ II absorption line systems and galaxies hosting $\mathrm{Mg}$ II-selected DLA absorbers.

We are however conscious that the stacking method may produce undesirable biases since it is unclear how some physical properties may be affected by this method. In Sects. 4 and 8.1, we have convincingly shown that the stacking method does not cause significant biases in representing the median value for the $\mathrm{O} / \mathrm{H}$ estimated values from the $R_{23}$ method, and for the extinction estimates, respectively. Both estimates are based on ratios of lines at different wavelengths. It is thus tempting to believe that temperatures, estimated in a similar way, would not be affected by the stacking method. However, we agree that this needs a full demonstration, which is beyond the scope of this paper. We also notice that the integrated spectrum of a single galaxy comes from all the contributed star-forming regions. By using stacked spectra from different galaxies, we are studying the resulted light coming from a larger set of star-forming regions. In principle our method is not less accurate than the determination of the $\mathrm{O} / \mathrm{H}$ through temperature for a single galaxy, and thus may not alter the calibrations proposed in this paper.

The major interest of the adopted stacked method presented here, is that it allows us for the first time to sample the stellar mass versus $\log (\mathrm{O} / \mathrm{H})$ relation using a temperature dependent method, up to relatively high stellar mass galaxies.

\subsection{The uncertainties of the $T([O \mathrm{II}] \lambda 7325)$-based abundances}

In the optical band, electron temperatures could usually be derived using five sets of auroral/nebular line intensity ratios: [O III $] \lambda 4363 /[\mathrm{O}$ III $] \lambda \lambda 4959,5007, \quad$ [S III] $] \lambda 6312 / \quad$ [S III] $] \lambda \lambda 9069$, 9532, [S II] $\lambda \lambda 6317,6731 /[\mathrm{S}$ II] $\lambda 4072$, [N II] $\lambda 5755 /[\mathrm{N}$ II] $\lambda \lambda 6548$, 6583, and [O II] $\lambda \lambda 7320,7330 /[\mathrm{O}$ II] $\lambda 3727$. However, for the integrated spectra of the SDSS sample galaxies with moderate stellar masses, only the indicator [O II $] \lambda \lambda 7320,7330 /[\mathrm{O}$ II $] \lambda 3727$ is available to derive their electron temperatures in the galactic gas since only [O II] $\lambda \lambda 7320,7330$ lines (marked as $\lambda 7325$ sometimes) are strong enough to be measured from these spectra. Therefore, the line ratio [O II] $\lambda \lambda 7320,7330 /[\mathrm{O}$ II $] \lambda 3727$ provides the only "direct" measurements for the electron temperature and the "direct" oxygen abundances for these moderate metal-rich galaxies, though some arguments exist to doubt the accuracy discussed as following.

One of the problems of $T(7325)$ is the contribution of recombination to the [O II $] \lambda 7325$ line. However, this has been estimated by KBG03 using the formula given by Liu et al. (2000), who found that recombination typically contributes less than $5 \%$ to the [O II] line flux, which corresponds to a temperature error of only $\sim 2-3 \%$ or less than $400 \mathrm{~K}$ for their worst case. A correct 
treatment of recombination should take into account the effect of temperature gradients within ionized nebulae.

The second problem is the inconsistency between the $T(7325)$ and the $T(5755)$ and $T(4072)$, presented by Bresolin et al. (2005). By using the VLT/FORS spectra, Bresolin et al. (2005) obtained the $T$ (7325) for $32 \mathrm{H}$ II regions located in some spiral galaxies, and $T(5755)$ and $T(6312)$ for only half of their sample since these two lines are much weaker. In addition, [S II]4072, from which $T$ (4072) was derived, has been seldom detected, since its measurement is made difficult by low $\mathrm{S} / \mathrm{N}$ ratio in the spectra. They compared these temperature values, and found that $T(7325)$ seems to overestimate the temperature if compared to $T(5755)$, while the opposite happens for $T$ (4072). However, the error-bars of the latter two temperatures are much larger than $T(7325)$, and these two lines are much weaker than $\lambda 7325$. We cannot measure the other temperaturesensitive auroral lines from most of our sample spectra.

Another problem is that the $T[\mathrm{O} I \mathrm{II}]$ vs. $T[\mathrm{O} \mathrm{III}]$ relation does not match the model fitting relation very well (see Fig. 1 of KBG03; Garnett 1992). They mentioned that the sources of this disagreement remain unresolved, but one of the sources may be the recombination contribution (Liu et al. 2000), others could be the radiative transfer effects and the observational uncertainties. However, their objects with large scatter mostly have $T$ [O III] between $9000 \mathrm{~K}$ and $12000 \mathrm{~K}$, and there may be a correlation between $T[\mathrm{O} \mathrm{II}]$ and $T[\mathrm{O} \mathrm{III}]$ in the extremes of the temperature range, e.g. $T_{\mathrm{e}}<9000 \mathrm{~K}$ and $T_{\mathrm{e}}>13000 \mathrm{~K}$. Most of our sample galaxies have just $T[\mathrm{O} \mathrm{III}]<8000 \mathrm{~K}$, thus no direct observations are against that our galaxies follow that correlation of $T[\mathrm{O} I \mathrm{II}] \mathrm{vs}$. $T[\mathrm{O}$ III] .

Therefore, the temperature derived from [O II] $\lambda 7325$ is acceptable, and is the only way to get the "direct" oxygen abundances for metal-rich galaxies.

\subsection{Temperature fluctuations and temperature gradients}

It has been argued for many years that directly measured electron temperatures and the corresponding abundances from collisionally excited lines may have systematic errors due to temperature fluctuations and temperature gradients, especially for the metalrich H II regions (Peimbert 1967; Stasinska 1980; Stasinska 2005). However, the auroral lines have been used to derive the "direct" electron temperature and the oxygen abundances for the metal-rich H II regions in recent years (KBG03; Garnett et al. 2004a,b; Bresolin et al. 2004, 2005; Bresolin 2007). KBG03 has discussed very clearly the "reliability" of the auroral line method for abundance estimates. We simply summarize some arguments and discussions presented by KBG03, Garnett (1992) and Stasinska (2005) regarding this topic.

In the presence of fluctuations in $T_{\mathrm{e}}$, forbidden line strengths can overestimate the average temperature, and therefore underestimate the true nebular abundances. However, the question of the existence of temperature fluctuations is still controversial (Peimbert 1967; Dinerstein et al. 1985; Shaver et al. 1983). The large-scale temperature gradient mimics the effects of temperature fluctuations, and can over-estimates the real temperature, and consequently the oxygen abundance is systematically underestimated (Garnett 1992; Stasinska 2005).

Despite these concerns, as KBG03 discussed, there are good reasons to believe that the forbidden lines give abundances that are close to correct, at least for the range of $\mathrm{O} / \mathrm{H}$ of interest here. Recent measurements of infrared forbidden lines (that have weak temperature dependences) in the spectra of planetary nebulae yield abundances in good agreement with those from optical forbidden lines, even where there is a large discrepancy with recombination line abundances (Liu et al. 2000, 2001). Likewise, measurements of radio recombination line temperatures of Galactic $\mathrm{H}$ II regions give results that are consistent with forbidden line measurements of the same objects (Shaver et al. 1983; Deharveng et al. 2000). In both cases, the line emissivity is only moderately dependent on $T_{\mathrm{e}}$. It would be worthwhile to explore the metal recombination lines and infrared fine-structure lines for the determination of electron temperature in the extragalactic $\mathrm{H}$ II regions of high metal content. However, there are obvious difficulties in exploiting these techniques: recombination lines are very weak and hard to detect in extragalactic H II regions (Esteban 2002), and infrared observations require orbiting telescopes (Garnett et al. 2004b).

\section{Conclusion}

We have derived direct oxygen abundances based on electron temperature for metal-rich galaxies, using an original method of stacking. To do so, we select a large sample of star-forming galaxies ( 23608) from the SDSS-DR4 and obtain 27 spectra by combining the spectra of the several hundred (even several thousand) galaxies within 27 various stellar mass bins from $\log \left(M_{*}\right)=8.0$ to 10.6 . This stacking method improves the $\mathrm{S} / \mathrm{N}$ ratios in a great deal of the spectra, without providing major biases. These high quality data with moderate resolution ( $3 \AA$ ) make it possible for us to measure the [O II] $\lambda \lambda 7320,7330$ emission-lines for the galaxies with stellar masses up to $\log \left(M_{*}\right) \sim 10.6$, then derive their electron temperature in the low ionization region by the ratio of $[\mathrm{O}$ II $] \lambda \lambda 7320,7330 /[\mathrm{O}$ II $] \lambda 3727$, hence the $T_{\mathrm{e}}$-based $\mathrm{O} / \mathrm{H}$ abundances.

It provides a new calibration of the $R_{23}$ method, which may well apply to galaxies with metallicities ranging from $12+\log (\mathrm{O} / \mathrm{H}) \sim 7.0$ to 9.0 . It also shows that the empirical $R_{23}$ calibrations overestimate the $\log (\mathrm{O} / \mathrm{H})$ abundances by about 0.2 to 0.6 dex. We also derive a new relationship between $T_{\mathrm{e}}$-derived $(\mathrm{O} / \mathrm{H})$ abundances and stellar masses from these moderate massive galaxies, which can be fitted by a linear fit, namely $12+\log (\mathrm{O} / \mathrm{H})=6.223+0.231 \times \log \left(M_{*}\right)$. The $T_{\mathrm{e}}$-based $\log (\mathrm{N} / \mathrm{O})$ abundance ratios show that their nitrogen sources are consistent with the combination of the primary and secondary components for these moderate metal-rich galaxies.

Acknowledgements. We especially thank our referee for the very valuable comments and suggestions, which helped greatly in improving this work. We thank Jing Wang and Grazyna Stasinska for helpful discussions about the underlying correction of stellar absorption for the SDSS spectra, thank Hong Wu, Jingyao $\mathrm{Hu}$, Licai Deng and Bo Zhang for interesting discussions. This work was supported by the Natural Science Foundation of China (NSFC) Foundation under Nos. 10403006, 10433010, 10673002, 10573022, 10333060, and 10521001; and the National Basic Research Program of China (973 Program) No. 2007CB815404. The STARLIGHT project is supported by the Brazilian agencies CNPq, CAPES and FAPESP and by the France-Brizil CAPES/Cofecub program.

\section{References}

Asari, N. V., et al. 2007, MNRAS, in press [arXiv:0707.3578]

Baldry, I. K., Glazebrook, K., Baugh, C. M., et al. 2002, ApJ, 569, 582

Bresolin, F., Garnett, D. R., \& Kennicutt, R. C. Jr. 2004, ApJ, 615, 228

Bresolin, F., Schaerer, D., Gonzàlez Delgado, R. M., \& Stasinska, G. 2005, A\&A, 441, 981

Bresolin, F. 2007, ApJ, 656, 186

Brinchmann, J., Charlot, S., White, S. D. M., et al. 2004, MNRAS, 351, 1151

Bruzual, A. G., \& Charlot, S. 2003, MNRAS, 344, 1000 (BC03)

Cardelli, J. A., Clayton, G. C., \& Mathis, J. S. 1989, ApJ, 345, 245 
Castellanos, M., Díaz, A. I., \& Terlevich, E. 2002, MNRAS, 337, 540 (CDT02) Chabrier, G. 2003, PASP, 115, 763

Charlot, S., \& Longhetti, M. 2001, MNRAS, 323, 887 (CL01)

Chang, R. X., Shen, S. Y., Hou, J. L., Shu, C. G., \& Shao, Z. Y. 2006, MNRAS, 372,199

Cid Fernandes, R., Mateus, A., Sodre, L., et al. 2005, MNRAS, 358, 363

Cid Fernandes, R., et al. 2007, Rev. Mex. Astron. Astrofis., in preperation de Robertis, M. M., Dufour, R. J., \& Hunt, R. W. 1987, JRASC, 81, 195 Denicoló, G., Terlevich, R., \& Terlevich, E. 2002, MNRAS, 330, 69 (D02)

Dinerstein, H. L., Lester, D. F., \& Werner, M. W. 1985, ApJ, 291, 561

Esteban, C., Peimbert, M., Torres-Peimbert, S., \& Rodríguez, M. 2002, ApJ, 581, 241

Garnett, D. R. 1992, AJ, 103, 1330

Garnett, D. R., Kennicutt, R. C. Jr., \& Bresolin, F. 2004a, ApJ, 607, L21

Garnett, D. R., Edmunds, M. G., Henry, R. B. C., Pagel, B. E. J., \& Skillman, E. D. 2004 b, AJ, 128,2722

Izotov, Y. I., Stasinska, G., Meynet, G., Guseva, N. G., \& Thuan, T. X. 2006, A\&A, 448, 955

Kauffmann, G., Heckman, T. M., Tremonti, C. A., et al. 2003, MNRAS, 346, 1055

Kennicutt, R. C. Jr., Bresolin, F., \& Garnett, D. R. 2003, ApJ, 591, 801 (KBG03)

Kewley, L. J., \& Dopita, M. A. 2002, ApJS, 142, 35

Kobulnicky, H. A., Kennicutt, R. C. Jr., \& Pizagno, J. L. 1999, ApJ, 514, 544 (K99)

Kobulnicky, H. A., \& Kewley, L. J. 2004, ApJ, 617, 240 (KK04)

Liang, Y. C., Yin, S. Y., Hammer, F., et al. 2006, ApJ, 652, 257

Liang, Y. C., \& Yin, S. Y. 2007, Proceedings of The central engine of Active Galactic Nuclei, ed. L. C. Ho, \& J.-M. Wang [arXiv: astro-ph/0701231]

Liu, X.-W., Luo, S.-G., Barlow, M. J., Danziger, I. J., \& Storey, P. J. 2001, MNRAS, 327, 141

Liu, X.-W., Storey, P. J., Barlow, M. J., et al. 2000, MNRAS, 312, 585

Mateus, A., Sodre, L., Cid Fernandes, R., et al. 2006, MNRAS, 370, 721
Mathis, H., Charlot, S., \& Brinchmann, J. 2006, MNRAS, 365, 385 McGaugh, S. S. 1991, ApJ, 380, 140

Pagel, B. E. J., Edmunds, M. G., Blackwell, D. E., et al. 1979, MNRAS, 189, 95 Peimbert, M. 1967, ApJ, 150, 825

Pettini, M., \& Pagel, B. E. J. 2004, MNRAS, 348, L59 (PP04)

Pilyugin, L. S. 2000, A\&A, 362, 325

Pilyugin, L. S. 2001a, A\&A, 369, 594 (P01)

Pilyugin, L. S. 2001b, A\&A, 373, 56 (P01)

Pilyugin, L. S., \& Thuan, T. X. 2005, ApJ, 631, 231 (PT05)

Pilyugin, L. S., Thuan, T. X., \& Vílchez, J. M. 2006, MNRAS, 367, 1139

Osterbrock, D. E. 1989, Astrophysics of Gaseous Nebulae and Active Galactic Nuclei., Mill Valley (California: University Science Books)

Salim, S., et al. 2007, ApJS, in press [arXiv:0704.3611]

Schiavon, R. P., Faber, S. M., Konidaris, N., et al. 2006, ApJ, 651, L93

Seaton, M. J. 1979, MNRAS, 187, 73

Shaver, P. A., McGee, R. X., Newton, L. M., Danks, A. C., \& Pottasch, S. R. 1983, MNRAS, 204, 53

Shaw, R. A., \& Dufour, R. J. 1995, PASP, 107, 896

Shi, F., Kong, X., \& Cheng, F. Z. 2006, A\&A, 453, 487

Stasińska, G. 1980, A\&A, 85, 359

Stasińska, G. 2005, A\&A, 434, 507

Stasińska, G. 2006, A\&A, 454, L127

Tremonti, C. A., Heckman, T. M., Kauffmann, G., et al. 2004, ApJ, 613, 898 (T04)

Yin, S. Y., Liang, Y. C., Hammer, F., et al. 2007a, A\&A, 462, 535 (Y07)

Yin, S. Y., Liang, Y. C., \& Zhang, B. 2007b, proceedings of The central engine of Active Galactic Nuclei, ed. L. C. Ho, \& J.-M. Wang [arXiv:astro-ph/0701234]

van Zee, L., \& Haynes, M. P. 2006, ApJ, 636, 214

Vila-Costas, M. B., \& Edmunds, M. G. 1993, MNRAS, 265, 119

Wild, V., Hewett, P. C., \& Pettini, M. 2007, MNRAS, 374, 292

Zheng, X. Z., Bell, E. F., Rix, H.-W., et al. 2006, ApJ, 640, 784 


\section{Online Material}


Y. C. Liang et al.: Oxygen abundances of metal-rich galaxies from $T_{\mathrm{e}}$, Online Material $p 2$
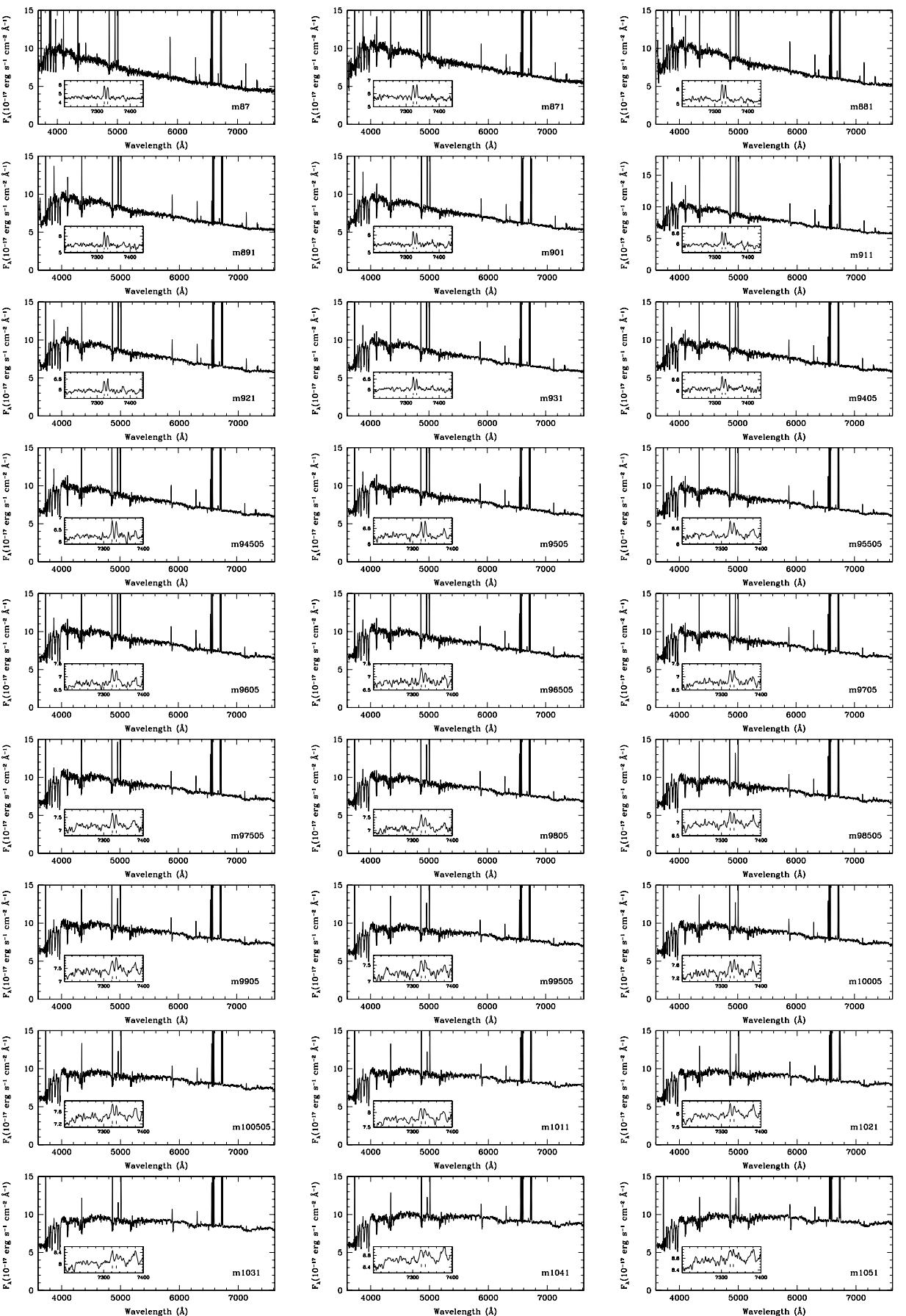

Fig. 1. The 27 combined spectra of the sample galaxies at rest-frame in the corresponding mass bins. The small figure inside each panel shows the auroral lines $[\mathrm{O}$ II] $\lambda \lambda 7320,7330$. The label for the mass bin of each spectrum is given in the bottom right corner. 
Y. C. Liang et al.: Oxygen abundances of metal-rich galaxies from $T_{\mathrm{e}}$, Online Material $p 3$

Table 2. The derived properties of the combined galaxies.

\begin{tabular}{|c|c|c|c|c|c|c|c|c|c|c|c|c|c|c|c|c|}
\hline$\overline{(1)}$ & $\overline{(2)}$ & (3) & $\overline{~(4)}$ & $\overline{~(5)}$ & $\overline{~(6) ~}$ & (7) & $(8)$ & $\overline{(9)}$ & $\overline{(10)}$ & $\overline{(11)}$ & $\overline{~(12) ~}$ & (13) & (14) & $\overline{~(15)}$ & $\overline{(16)}$ & $\overline{~(17) ~}$ \\
\hline mass & s2 ratio & $n_{e}\left(\mathrm{~cm}^{-3}\right)$ & $\mathrm{o} 2$ ratio & $T_{\mathrm{e}}([\mathrm{O} \mathrm{II}])(\mathrm{K})$ & $T_{\mathrm{e}}([\mathrm{O} \mathrm{III}])(\mathrm{K})$ & $\mathrm{OH}_{T_{\mathrm{e}}}$ & $A_{V}$ & $\log R_{23}$ & $\log \mathrm{O}_{32}$ & $\mathrm{OH}_{R_{23}}^{a}$ & $\mathrm{OH}_{R_{23}}^{b}$ & $P$ & $\mathrm{OH}_{P}$ & $N 2$ & $\mathrm{OH}_{N 2}$ & $\log \left(\frac{N}{O}\right)$ \\
\hline$\overline{8.70}$ & 1.36 & 58.97 & $44.25 \pm 0.95$ & $11423 \pm 153$ & $12034 \pm 219$ & $8.05 \pm 0.03$ & 0.18 & 0.77 & 0.08 & 8.48 & 8.63 & 0.54 & 8.39 & -1.08 & 8.28 & -1.29 \\
\hline 8.71 & 1.38 & 39.36 & $51.32 \pm 2.76$ & $10641 \pm 341$ & $10916 \pm 487$ & $8.23 \pm 0.07$ & 0.42 & 0.82 & -0.05 & 8.34 & 8.58 & 0.47 & 8.29 & -0.94 & 8.36 & -1.31 \\
\hline 8.81 & 1.43 & 3.58 & $47.38 \pm 2.39$ & $11491 \pm 370$ & $12130 \pm 528$ & $8.06 \pm 0.07$ & 0.38 & 0.78 & -0.11 & 8.47 & 8.63 & 0.44 & 8.31 & -0.94 & 8.36 & -1.24 \\
\hline 8.91 & 1.41 & 14.06 & $63.38 \pm 0.29$ & $9703 \pm 22$ & $9576 \pm 32$ & $8.35 \pm 0.01$ & 0.35 & 0.75 & -0.21 & 8.56 & 8.67 & 0.38 & 8.32 & -0.88 & 8.40 & -1.31 \\
\hline 9.01 & 1.40 & 24.83 & $62.37 \pm 2.23$ & $9701 \pm 182$ & $9572 \pm 260$ & $8.36 \pm 0.05$ & 0.39 & 0.75 & -0.22 & 8.54 & 8.66 & 0.38 & 8.31 & -0.86 & 8.41 & -1.29 \\
\hline 9.11 & 1.37 & 50.84 & $64.91 \pm 2.75$ & $9328 \pm 202$ & $9039 \pm 289$ & $8.41 \pm 0.06$ & 0.36 & 0.72 & -0.24 & 8.62 & 8.70 & 0.36 & 8.34 & -0.80 & 8.44 & -1.25 \\
\hline 9.21 & 1.39 & 33.32 & $69.04 \pm 0.80$ & $9168 \pm 51$ & $8812 \pm 72$ & $8.44 \pm 0.02$ & 0.41 & 0.71 & -0.28 & 8.64 & 8.71 & 0.34 & 8.34 & -0.77 & 8.46 & -1.23 \\
\hline 9.31 & 1.38 & 40.24 & $69.45 \pm 0.39$ & $9096 \pm 24$ & $8709 \pm 34$ & $8.43 \pm 0.01$ & 0.40 & 0.68 & -0.31 & 8.70 & 8.75 & 0.33 & 8.36 & -0.74 & 8.48 & -1.19 \\
\hline 9.405 & 1.36 & 57.15 & $63.03 \pm 0.50$ & $9422 \pm 38$ & $9174 \pm 54$ & $8.35 \pm 0.01$ & 0.47 & 0.66 & -0.36 & 8.73 & 8.77 & 0.30 & 8.37 & -0.72 & 8.49 & -1.14 \\
\hline 9.4505 & 1.39 & 37.63 & $73.34 \pm 1.29$ & $8883 \pm 74$ & $8404 \pm 105$ & $8.48 \pm 0.02$ & 0.53 & 0.68 & -0.39 & 8.71 & 8.75 & 0.29 & 8.35 & -0.69 & 8.50 & -1.18 \\
\hline 9.505 & 1.42 & 13.25 & $92.13 \pm 0.91$ & $8169 \pm 34$ & $7384 \pm 49$ & $8.67 \pm 0.01$ & 0.59 & 0.67 & -0.39 & 8.73 & 8.77 & 0.29 & 8.36 & -0.70 & 8.50 & -1.25 \\
\hline 9.5505 & 1.42 & 10.81 & $76.59 \pm 2.96$ & $8878 \pm 164$ & $8397 \pm 235$ & $8.47 \pm 0.05$ & 0.59 & 0.66 & -0.40 & 8.73 & 8.77 & 0.29 & 8.36 & -0.67 & 8.52 & -1.14 \\
\hline 9.605 & 1.40 & 29.90 & $75.72 \pm 0.09$ & $8801 \pm 5$ & $8287 \pm 7$ & $8.46 \pm 0.00$ & 0.54 & 0.63 & -0.42 & 8.80 & 8.81 & 0.27 & 8.40 & -0.63 & 8.54 & -1.09 \\
\hline 9.6505 & 1.38 & 41.11 & $79.67 \pm 0.77$ & $8534 \pm 37$ & $7906 \pm 53$ & $8.52 \pm 0.01$ & 0.67 & 0.62 & -0.46 & 8.81 & 8.82 & 0.26 & 8.40 & -0.63 & 8.54 & -1.11 \\
\hline 9.705 & 1.39 & 32.46 & $83.36 \pm 1.99$ & $8414 \pm 90$ & $7735 \pm 128$ & $8.55 \pm 0.03$ & 0.66 & 0.62 & -0.45 & 8.81 & 8.82 & 0.26 & 8.40 & -0.62 & 8.55 & -1.11 \\
\hline 9.7505 & 1.38 & 39.36 & $77.80 \pm 1.99$ & $8636 \pm 102$ & $8051 \pm 146$ & $8.47 \pm 0.03$ & 0.68 & 0.59 & -0.50 & 8.84 & 8.84 & 0.24 & 8.42 & -0.59 & 8.57 & -1.04 \\
\hline 9.805 & 1.38 & 39.36 & $76.95 \pm 1.50$ & $8679 \pm 75$ & $8112 \pm 108$ & $8.42 \pm 0.03$ & 0.74 & 0.55 & -0.50 & 8.89 & 8.88 & 0.24 & 8.45 & -0.58 & 8.57 & -0.99 \\
\hline 9.8505 & 1.38 & 42.86 & $89.25 \pm 1.48$ & $8114 \pm 58$ & $7305 \pm 83$ & $8.59 \pm 0.02$ & 0.77 & 0.57 & -0.54 & 8.88 & 8.87 & 0.22 & 8.43 & -0.56 & 8.58 & -1.05 \\
\hline 9.905 & 1.39 & 32.46 & $69.83 \pm 2.42$ & $9124 \pm 156$ & $8748 \pm 224$ & $8.29 \pm 0.05$ & 0.80 & 0.53 & -0.54 & 8.91 & 8.89 & 0.23 & 8.47 & -0.56 & 8.58 & -0.91 \\
\hline 9.9505 & 1.36 & 64.48 & $66.14 \pm 2.55$ & $9156 \pm 177$ & $8794 \pm 253$ & $8.28 \pm 0.05$ & 0.83 & 0.53 & -0.55 & 8.92 & 8.90 & 0.22 & 8.47 & -0.53 & 8.60 & -0.88 \\
\hline 10.005 & 1.37 & 54.43 & $88.12 \pm 1.56$ & $8096 \pm 62$ & $7281 \pm 88$ & $8.55 \pm 0.02$ & 0.81 & 0.52 & -0.56 & 8.92 & 8.91 & 0.21 & 8.48 & -0.52 & 8.60 & -0.97 \\
\hline 10.0505 & 1.38 & 40.24 & $98.15 \pm 6.10$ & $7813 \pm 210$ & $6876 \pm 299$ & $8.63 \pm 0.09$ & 0.87 & 0.51 & -0.56 & 8.93 & 8.92 & 0.22 & 8.49 & -0.50 & 8.61 & -0.97 \\
\hline 10.11 & 1.34 & 76.67 & $76.26 \pm 1.60$ & $8563 \pm 82$ & $7947 \pm 118$ & $8.39 \pm 0.03$ & 0.94 & 0.49 & -0.58 & 8.95 & 8.93 & 0.21 & 8.50 & -0.49 & 8.62 & -0.87 \\
\hline 10.21 & 1.34 & 75.72 & $101.92 \pm 2.07$ & $7531 \pm 61$ & $6472 \pm 88$ & $8.70 \pm 0.03$ & 1.06 & 0.48 & -0.61 & 8.95 & 8.94 & 0.20 & 8.50 & -0.47 & 8.63 & -0.96 \\
\hline 10.31 & 1.32 & 97.12 & $97.23 \pm 2.54$ & $7579 \pm 77$ & $6542 \pm 110$ & $8.66 \pm 0.04$ & 1.08 & 0.46 & -0.61 & 8.97 & 8.96 & 0.20 & 8.52 & -0.46 & 8.64 & -0.92 \\
\hline 10.41 & 1.33 & 86.29 & $81.74 \pm 1.54$ & $8197 \pm 68$ & $7424 \pm 97$ & $8.45 \pm 0.03$ & 1.15 & 0.45 & -0.60 & 8.97 & 8.96 & 0.20 & 8.53 & -0.44 & 8.65 & -0.81 \\
\hline 10.51 & 1.36 & 57.15 & $110.86 \pm 1.85$ & $7368 \pm 48$ & $6241 \pm 69$ & $8.76 \pm 0.02$ & 1.22 & 0.48 & -0.60 & 8.95 & 8.94 & 0.20 & 8.50 & -0.44 & 8.65 & -0.95 \\
\hline
\end{tabular}

Notes: Columns (1)-(17) consequently refer to: (1) the mass of the center value of the bin; (2) the ratio of [S II] $] 6717 /[\mathrm{S}$ II] $] \lambda 6731$; (3) the electron density; (4) the ratio of $[\mathrm{O} \mathrm{II}] \lambda \lambda 7320,7330 /[\mathrm{O}$ II $] \lambda 3727$; (5) the election temperature in low ionization region; (6) the election temperature in high ionization region; $(7)$ the oxygen abundances $12+\log (\mathrm{O} / \mathrm{H})$ derived from election temprature; (8) the dust extinction $A_{V}$ estimated from $\mathrm{H} \alpha / \mathrm{H} \beta$; (9) logarithm of the ratio of ([O II $] \lambda 3727+[\mathrm{O}$ III] $] \lambda \lambda 4959$, 5007)/H $\beta$; (10) logarithm of the ratio of [O III] $] \lambda \lambda 4959,5007 /[\mathrm{O}$ II $] \lambda 3727 ;(11)$ the $12+\log (\mathrm{O} / \mathrm{H})$ abundances obtained by using the $R_{23}$ calibrations derived from DR4 (Eq. (2)); (12) the $12+\log (\mathrm{O} / \mathrm{H})$ abundances obtained by using the $R_{23}$ calibrations derived from DR2 (T04); (13) the $P$ parameter [O III $\left.] \lambda \lambda 4959,5007\right) /([\mathrm{O}$ II $] \lambda 3727+[\mathrm{O}$ III $] \lambda \lambda 4959,5007) ;(14)$ the $12+\log (\mathrm{O} / \mathrm{H})$ abundances obtained by using the $P$-method calibration of P01; (15) the $N 2$ index $(=\log ([\mathrm{N}$ II $] \lambda 6583 / \mathrm{H} \alpha)) ;(16)$ the $12+\log (\mathrm{O} / \mathrm{H})$ abundances obtained by using the $N 2$ calibration of PP04; (17) the $\log (\mathrm{N} / \mathrm{O})$ abundance ratios derived from election temperature method. 